\title{
A race between Proactive and Reactive processes during perceptual decisions
}

\author{
Lluís Hernández-Navarro ${ }^{1}$, Ainhoa Hermoso-Mendizabal ${ }^{1}$, Daniel Duque ${ }^{1}$, Jaime de la \\ Rocha $^{1, *}$ and Alexandre Hyafil ${ }^{2,3, *, *}$
}

\author{
${ }^{1}$ IDIBAPS, Rosselló 149, Barcelona, 08036, Spain \\ ${ }^{2}$ Center for Brain and Cognition, Universitat Pompeu Fabra, Ramón Trias Fargas, 25, 08018 \\ Barcelona, Spain \\ ${ }^{3}$ Center of Mathematical Research, Campus UAB Edifici C, Bellaterra, 08193, Spain \\ * Correspondence: alexandre.hyafil@gmail.com \\ * Equal contributions
}

\begin{abstract}
Standard models of perceptual decision-making postulate that a response is triggered in reaction to stimulus presentation when the accumulated stimulus evidence reaches a decision threshold. This framework excludes however the possibility that informed responses are generated proactively at a time independent of stimulus. Here, we find that, in a free reaction time auditory task in rats, reactive and proactive responses coexist, suggesting that choice selection and motor initiation, commonly viewed as serial processes, are decoupled in general. We capture this behavior by a novel model in which proactive and reactive responses are triggered whenever either of two competing processes, respectively Action Initiation or Evidence Accumulation, reaches a bound. In both types of response, the choice is ultimately informed by the Evidence Accumulation process. By including the Action Initiation process, the model readily explains premature responses, urgency effects at long reaction times and the slowing of the responses as animals get satiated and tired during sessions. Moreover, it successfully predicts reaction time distributions when the stimulus was either delayed, advanced or omitted. Overall, these results fundamentally extend standard models of evidence accumulation in decision making by showing that proactive and reactive processes compete for the generation of responses.
\end{abstract}

\section{INTRODUCTION}

Our brains must constantly make perceptual decisions in the face of ambiguous and noisy stimuli. A very successful framework in cognitive psychology and neuroscience suggests that, under free response conditions, these perceptual decisions follow an accumulation to bound policy: sensory evidence is integrated over time until a given decision criterion is met (Wald, 1947; Good, 1979; Gold and Shadlen, 2002). Computational models implementing this idea, such as the Drift-Diffusion Model (DDM) (Ratcliff, 1978; Bogacz et al., 2006; 
Ratcliff and McKoon, 2008) and race models (Pike, 1971, 1973), provide a good quantitative account for reaction times (RTs) and choices in humans (Milosavljevic et al., no date; Becker, 1958; Ratcliff and Smith, 2004; Gold and Shadlen, 2007; Simen et al., 2009; Brunton, Botvinick and Brody, 2013; Ratcliff et al., 2016; Hanks and Summerfield, 2017; Shevinsky and Reinagel, 2019), non-human primates (Gold and Shadlen, 2001, 2007; Ratcliff et al., 2016; Hanks and Summerfield, 2017), and rats (Pardo-Vazquez et al. 2019). A key assumption of the DDM and other accumulation models is that decision-making is essentially a serial process where the motor response is initiated reactively, only once the evidence accumulation has reached the decision boundary. Hence, choices and RTs are intrinsically coupled.

In real life, however, motor actions are not always triggered by a stimulus or external event. Proactive responses are at play in self-paced actions in the absence of stimuli (Romo and Schultz, 1992; Lee and Assad, 2003; Murakami et al., 2014), like in foraging decisions (Jung et al., 2014), but they can also be particularly prevalent in sensory driven decisions when the stimulus onset can be anticipated (Green, Smith and von Gierke, 1983; Carpenter and Williams, 1995) or under strong time pressure (Stanford et al., 2010; Salinas et al., 2014; Haith, Pakpoor and Krakauer, 2016; Hardwick et al., 2019). Moreover, even when subjects accumulate a fixed amount of sensory evidence before eliciting a response, reaching this criterion may at times take too long (Churchland, Kiani and Shadlen, 2008; Drugowitsch et al., 2012; Thura et al., 2012). In those circumstances, subjects may need to proactively terminate the accumulation process and trigger a response independently of the accumulated evidence. Therefore, although previous studies characterizing proactive and reactive responses have implicitly assumed that they represent distinct response modes deployed in different contexts, the two may actually coexist. The possible coexistence of proactive and reactive processes remains unknown, and so are the mechanisms describing their possible interactions.

To address these questions, we analysed the RTs and choices of rats in an auditory discrimination task (Hermoso-Mendizabal et al., 2020). Our results show that, in addition to reactive responses triggered by sensory evidence accumulation, animals exhibit informed proactive responses whose timing is independent of the stimulus. This coexistence was captured by a novel model of perceptual decision-making, whereby responses can be triggered by either of two parallel dynamical processes: Action Initiation, yielding proactive responses; or Evidence Accumulation, yielding reactive responses. The model correctly captured reaction times distributions and choices, and their dependence on both stimulus characteristics as well as internal variables such as satiety and fatigue. Overall, these results extend standard models of decision-making by showing that, in freely-timed paradigms, both evidence accumulation and action initiation run in parallel and compete with each other to be the triggers of our actions.

\section{RESULTS}

\section{Reaction time auditory task}


We trained rats in two variants of a reaction-time auditory discrimination task (Hermoso-Mendizabal et al., 2020) (Fig. 1). On each variant, an acoustic stimulus was played after a $300 \mathrm{~ms}$ fixation period at the center port, and interrupted once the animal withdrew from the center port to make a response by poking at one of the two side ports. Since the fixation period was constant, animals could predict the time of the stimulus onset. Withdrawal of the center port before stimulus onset, i.e. fixation breaks (FB), led to trial abortion. The stimulus was a superposition of two amplitude-modulated (AM) sounds and the animals were rewarded for correctly discriminating the sound with the higher average intensity. The two sounds were either two pure tones with different frequency (frequency discrimination task, Group 1, $n=10$ ) or two noise bursts coming from the left and the right speaker (laterality discrimination task, for Groups $2-4, n=16$ ). The discrimination difficulty of each stimulus (i.e. stimulus strength $s$ ) set the relative amplitude of each sound. Stimulus sequences included serial correlations to study expectation-mediated choice biases (see Supp. Methods). For our current analysis, we focus however on trials following an error, where we have previously shown that animals do not leverage on the serial correlations to bias their choices (unbiased trials) (Hermoso-Mendizabal et al., 2020) (Supplementary Fig. 1). The results obtained in this condition also held for expectation-biased trials (see below).

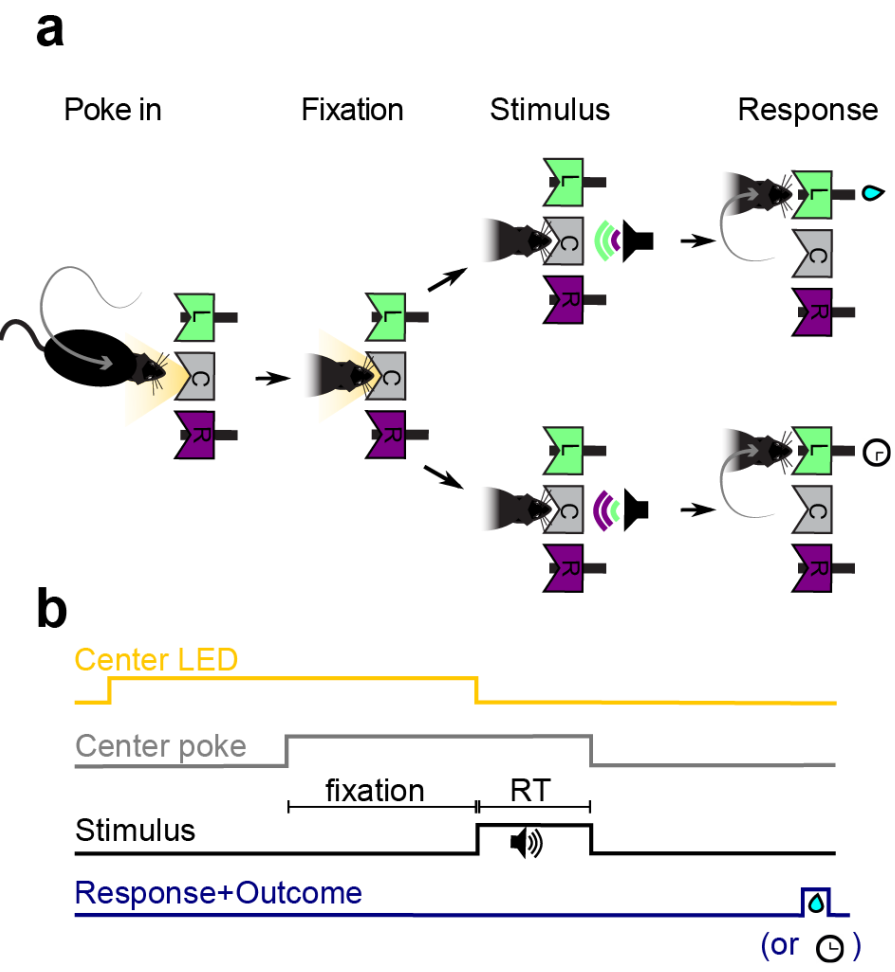

Fig. 1 Auditory discrimination task. a, The initiation of each trial was cued by a center port LED. Following the cue, rats poked in the center port and the fixation period $(300 \mathrm{~ms})$ started. At the end of the fixation period, the stimulus was presented, consisting in a mixture of two AM sounds, each of which is associated with reward in the Left $(L)$ or Right $(R)$ port. The two sounds differed in frequency (frequency discrimination task) or in the location of the sound source (lateralization discrimination task). After stimulus onset, the rats were free to withdraw from the center port to elicit a response, causing the stimulus to stop. Correct responses were rewarded with water and incorrect responses were punished by a time-out. b, Task temporal scheme: RT denotes the time from stimulus onset to center poke withdrawal. 


\section{Decoupling of reaction times and choices}

Standard models of evidence-to-bound integration predict that, as the stimulus strength increases, accuracy also increases, while reaction time (RT) decreases (Ratcliff, 1978; Bogacz et al., 2006; Ratcliff and McKoon, 2008). As expected, in the frequency discrimination task, RTs did indeed decrease with stimulus strength $s$, indicating that at least a fraction of responses were reactive, although the overall mean modulation was weak ( $2 \%$ FOV explained). Surprisingly however, RTs shorter than $\sim 90 \mathrm{~ms}$ were independent of stimulus strength (Fig. 2a). To assess more precisely how stimulus strength modulates RTs, we computed time delay curves, which measured the advancement of responses below a given RT as stimulus strength increased (Fig. 2b; Methods and Supplementary Fig. 2) (Pardo-Vazquez et al., 2019). The time beyond which RTs showed a significant modulation with $s$ was consistent across rats (mean stimulus modulation onset $\mathrm{M}=95 \mathrm{~ms}, \mathrm{SD}=20 \mathrm{~ms}$; vertical line in Fig. 2b). We called express responses (Carpenter and Williams, 1995) the responses occurring between stimulus onset and this modulation onset, which accounted for $35 \%(S D=25 \%)$ of all responses. We wondered whether express responses corresponded to rushed responses in which rats did not have time to process the stimulus, and made a guess choice based on stimulus-independent factors. We found however, that categorization accuracy for express responses was well above chance and increased with stimulus strength in all animals, demonstrating that rats used the stimulus to determine their choice even in express responses (Fig. 2c). This dependence was well illustrated by the tachometric curve, representing how choice accuracy varies with RT (Fig. 2d) (Stanford et al., 2010). During the interval of express responses, accuracy increased, until it reached a maximum and a relative plateau for non-express responses. These results also hold for both expectation-biased trials and for the laterality version of the task (Supplementary Figs. 3 and 9).

Overall, the timing of rat responses seemed to follow two modes: express responses, where the timing was independent of evidence accumulation but the choice did depend on the stimulus; and slower responses, where both the timing and the choice accuracy depend on the stimulus. Express responses are incompatible with standard models of evidence accumulation and their extensions, because these models inherently rely on evidence bounding to trigger the response and therefore, they invariably predict a coupling between RT and stimulus strength for all responses (Bowman, Kording and Gottfried, 2012; Drugowitsch et al., 2012; Thura et al., 2012). Slower responses in contrast, could in principle be triggered by the same evidence accumulation process giving rise to choice selection, and thus seem compatible with standard models of evidence accumulation. Because of this dichotomy, we next developed a general model for perceptual decision-making that could account for the full spectrum of rats' responses found in our task. 

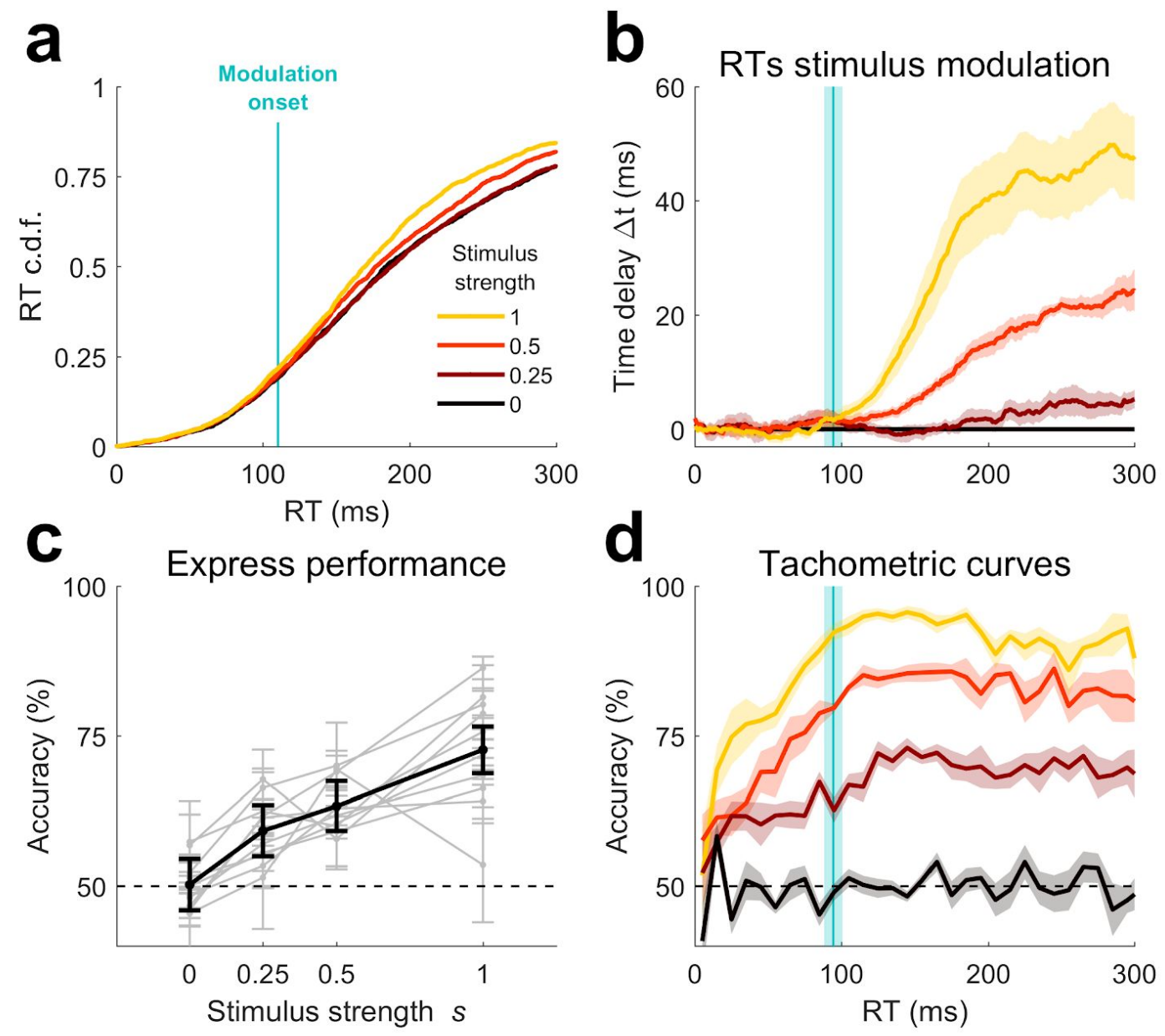

Fig. 2 | Decoupled reaction times and choices for express responses. a, RT cumulative distribution function (cdf) for different values of stimulus strength for an example animal (rat \#11) in unbiased trials. Vertical line indicates the onset of the stimulus modulation on RT cdf computed for this rat (see Methods). b, Mean time delay curves for RT cdf in the frequency discrimination task, averaged across rats (Group 1, $n=10$ ). Trials with uninformative stimulus (stim. strength $s=0$ ) are used as reference (time delay $\Delta t=0$ ). Vertical line represents the mean modulation onset time (95 ms). Shaded areas: standard error of the mean (s.e.m). c, Accuracy on trials with RTs shorter than 50 $\mathrm{ms}$ as a function of stimulus strength $s$. Thick line denotes the group average; thin lines represent individual rats. Error bars represent s.e.m. d, Tachometric curves, i.e. accuracy as a function of reaction times (Stanford et al., 2010), for different stimulus strength $s$ values, averaged across rats. Reaction times were binned in windows of $10 \mathrm{~ms}$. Vertical line: mean RT-stimulus modulation onset. Shaded areas: s.e.m.

\section{A parallel model of action initiation and sensory evidence integration captures RT-stimulus decoupling}

To capture the partial stimulus-independence of rat reaction times, we introduced a novel model, the Parallel Sensory Integration and Action Model (PSIAM) composed of: (1) a standard Evidence Accumulation process (EA) that integrates stimulus evidence over time and that is bounded by left and right decision bounds, i.e. a standard DDM (Fig. 3b, top) (Ratcliff, 1978; Bogacz et al., 2006; Ratcliff and McKoon, 2008); (2) an independent Action Initiation process $(\mathrm{Al})$ which reflects the preparation of a response. Because the fixation 
period preceding the stimulus was fixed, rats could prepare the motor action during this period in order to respond rapidly after stimulus onset, while maintaining a reasonable accuracy. The Al process represented such proactive timing signal (Simen et al., 2011), and was implemented as a one-bounded diffusive process that ramped until it reached the Go bound, independently of stimulus. The PSIAM can generate two types of responses, proactive or reactive, depending on which of the two processes, the Al or the EA, reaches a bound first. If the Al process hits the Go bound first (proactive response; Fig. 3a, bottom) a response is triggered, and the choice is given by the sign of the total accumulated evidence, i.e. the sign of the EA process (Fig. 3a, top). This procedure is equivalent to an instantaneous collapse of EA bounds at the time of response (Fig. 3a). In proactive responses, the timing of the response is thus completely independent of the stimulus strength. Conversely, if EA reaches a decision bound first (reactive response), it sets both RT and choice, and Al plays no role, as in a standard DDM (Fig. 3b). In this framework, express responses correspond to proactive responses that took place before the EA could start triggering any response: this time corresponds to the sum of EA latency $t_{\mathrm{E}}$ and the minimal EA integration-to-boundary time (Fig. 3a).

We assessed the capacity of the PSIAM to quantitatively capture rat behavior by fitting the model to the RTs. The model nicely captured the shape of the RT distributions for each animal by identifying the two underlying components, i.e. the proactive and reactive responses (Figs. 3c; Supplementary Fig. 4). This separation allowed the model to readily account for the fixation breaks (FBs), interpreted as proactive responses occurring before stimulus onset (Fig. 3c). The model also captured how the upward deflection in the RT distributions, reflecting the earliest reactive responses, increased with stimulus strength (Fig. 3c; Supplementary Fig. 4, last column). The agreement between data and model in the RT pdfs is also visible in the time delay curves, which, as previously shown for the data (Fig. 2b), were all collapsed for express RTs, and displayed increasing modulation by stimulus strength afterwards (Fig. 3d). Furthermore, the model also captured the shape of the experimental tachometric curves (Fig. 3e). Note that animal choices were not used to estimate the parameters of the model, so the tachometric curves are direct predictions from the model (see Methods). The model captured the increase in accuracy for fast RTs (RT<100 ms), followed by a relatively flat plateau for intermediate RTs (from 100 to $300 \mathrm{~ms}$ ). More importantly, the model provided an explanation for the shape of the tachometric curves: for express responses, where the time of integration of the EA process is set by the Al process, longer RTs allow for longer integration of stimulus evidence, leading to higher accuracy. Intermediate RTs were dominated on the other hand by reactive responses caused by the EA reaching a decision bound. For such responses, the PSIAM is equivalent to the standard DDM with constant bounds, where the accuracy is independent of RT (Ratcliff, 1978; Bogacz et al., 2006; Ratcliff and McKoon, 2008). This accounts for the relatively flat tachometric curves at each stimulus strength $s$ for these intermediate RTs. Of note, the PSIAM also captured the RT distributions and reproduced the time delay and tachometric curves for biased trials (Supplementary Figs. 5 and 6). Overall, the PSIAM successfully captured rats' complex patterns of RTs and choices by stipulating that response times were controlled by parallel, independent evidence accumulation and action initiation processes. 

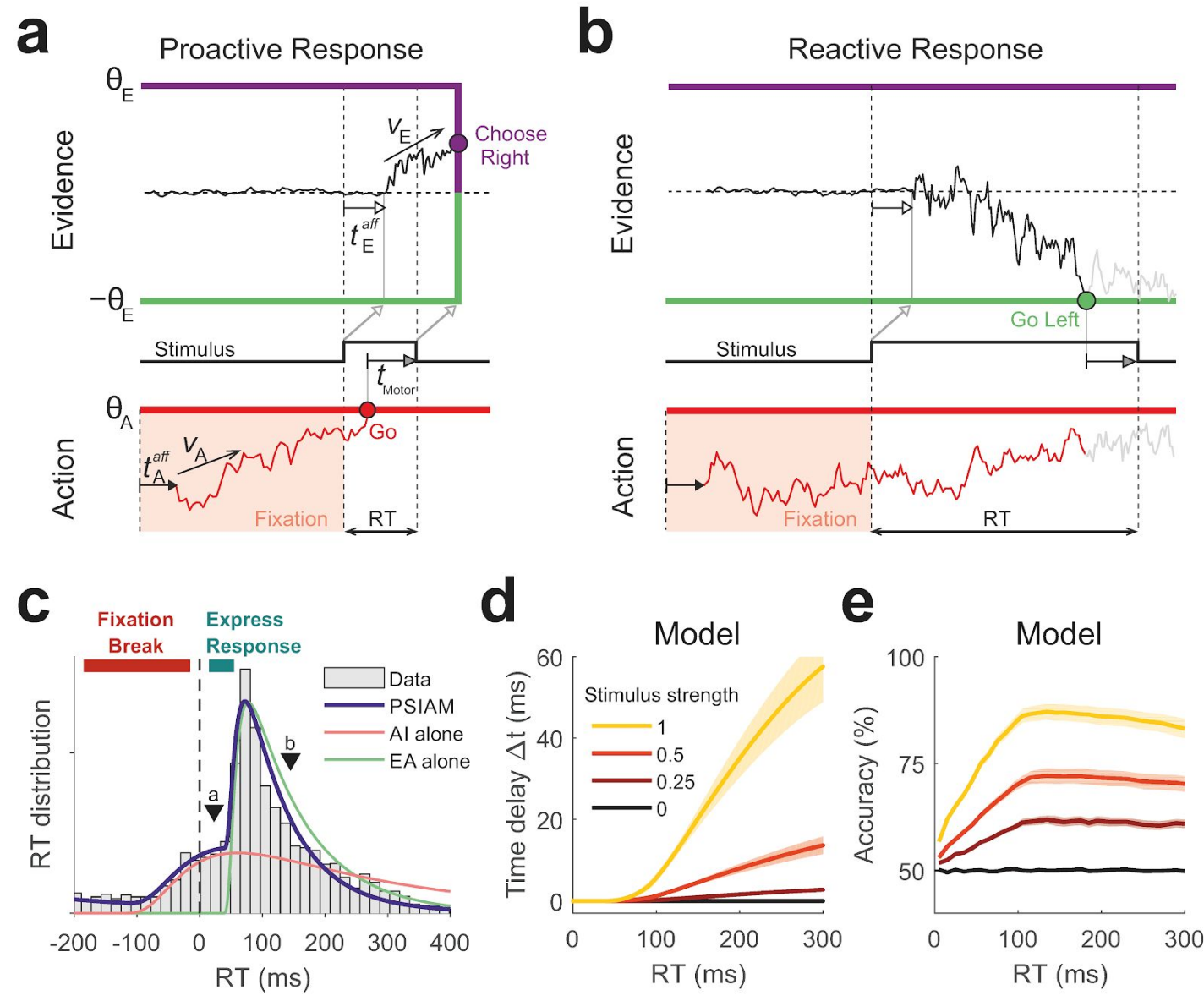

Fig. 3 | Parallel Sensory Integration and Action model. a, Example of a proactive response generated by the PSIAM. The action initiation process (AI, red trace) starts ramping up with afferent latency $t_{\mathrm{A}}^{\text {aff }}$ after the fixation onset, with mean drift $v_{A}$, until it hits the Go boundary $\theta_{A}$. The evidence accumulation process (EA, black trace) starts in response to the stimulus onset and after an afferent, sensory latency $t_{E}^{\text {aff }}$, with a stimulus-dependent mean drift $v_{E}$, and symmetric decision bounds $\pm \theta_{E}$. Reaction time is determined by the first of the $\mathrm{Al}$ and EA processes reaching a bound. For this example, Al reaches the Go bound first, triggering a proactive response after a motor latency $t_{\text {motor }}$. The stimulus is stopped at the response time (middle scheme). The choice (Right) is then determined by the final sign of the EA process, after the full stimulus has been integrated. This read-out is illustrated by an instantaneous collapse of the decision bounds. $\mathbf{b}$, Example of a reactive response, where the EA process reaches a decision bound first, which triggers a response after the motor latency $t_{\text {motor, }}$ setting both RT and choice; the Al process plays no role. Because from the behavioral perspective the actual latency is only the sum of afferent and motor latencies, we define for each process the effective latencies $t_{A}=t_{A}^{\text {aff }}+t_{\text {motor }}$ and $t_{E}=t_{E}^{\text {aff }}+t_{\text {motor }} \mathbf{c}$, RT distribution for an example rat (gray bars; rat \#12) and model fit (blue line) for maximum stimulus strength $s=1$. The distributions of proactive (red) and reactive (green) RTs generated by the Al and EA processes working in isolation are also shown. d, Simulated time delay curves, generated from the PSIAM fit to RTs for each animal, averaged across rats (Group 1, $n=10$ ). Shaded areas: s.e.m. e, Simulated tachometric curves, predicted from fitting the PSIAM to each animal's RTs, averaged across rats. RT bin width is $10 \mathrm{~ms}$; shaded areas: s.e.m.

\section{Proactive responses prevent longer reaction times}


While our Al process can be thought of as an independent urgency signal, urgency signals have been mostly hypothesized to play a role in avoiding very long reaction times, rather than promoting very fast responses (Drugowitsch et al., 2012; Thura et al., 2012). We thus wondered whether the Al could play a role beyond express responses, at long RTs. By construction, PSIAM responses are faster when both EA and Al processes are taken into account than when EA is considered in isolation (Fig. 4a, blue, green and red curves, respectively; Supplementary Fig. 7). Proactive responses dominated fast RTs, whereas reactive responses took over for intermediate RTs; however, proactive responses contributed as much as reactive responses at long RTs (Fig. 4b; Supplementary Fig. 8). These long proactive responses imply that, in those trials, the EA process had still not reached the decision bound, and thus the Al process acted as an urgency signal that prevented very slow reactive responses. Moreover, because in those trials the evidence level is lower than the decision bound, their associated accuracy should also be lower than for reactive choices with the same RT. In other words, the increase in the fraction of proactive responses should thus be accompanied by a reduction in categorization accuracy. We tested this prediction by extending the tachometric curves to these very long, infrequent RTs. As predicted by the PSIAM, we found a significant decrease in accuracy for long RTs (Fig. 4c-d). In sum, Al did not only promote express responses but it also shaped slow responses, hindering their occurrence at the expense of reduced accuracy for long RTs.
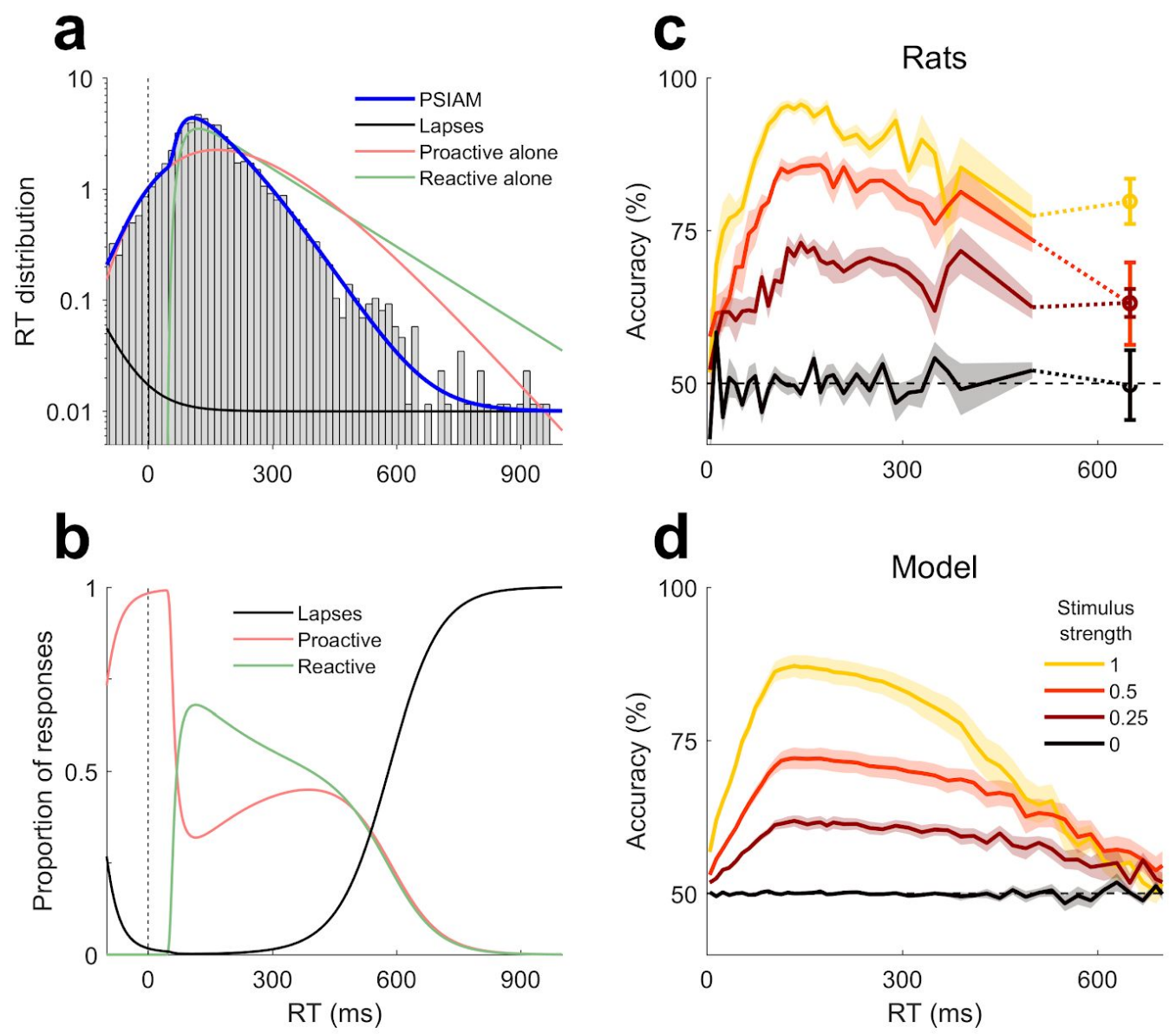

Fig. 4 Reaction times and accuracy for long responses. a, RT distribution for an example rat (gray bars) and model fit (blue line) for medium stimulus strength $(s=0.5)$. To better resolve the tail of 
the distribution we used a logarithmic scale. Notice how the tail of the PSIAM distribution has been substantially trimmed with respect to the distributions of proactive alone (red) and reactive alone (green) RTs generated by the Al and EA processes working in isolation. For very long RTs (above 600 $\mathrm{ms}$ ), lapse responses, modelled as random guesses, dominate the pdf (black curve; average $7 \pm 3 \%$ of the total responses; see Methods and Supplementary Fig. 7). Distribution of lapse RTs is shown in black. b, Proportion of proactive, reactive and lapse responses vs. RT for the same example rat as in a. c, Tachometric curves for different stimulus strength $s$ values, averaged across rats. Open circles indicate accuracy for responses of RT $>600$ ms. Shaded areas and error bars: s.e.m. d, Simulated tachometric curves up to $700 \mathrm{~ms}$, averaged across rats; shaded areas: s.e.m.

\section{Manipulating stimulus presentation uncovers proactive and reactive responses}

The PSIAM core hypothesis is that, on each trial, animals prepare a proactive response which is executed independently of the presentation of the stimulus, unless a reactive response is triggered first. The model then predicts that, if the stimulus is removed, animal responses should all be proactive responses triggered by the Al process. We tested this in a new group of rats (Group 2, $n=6$; laterality discrimination task) where we omitted the presentation of the stimulus in a subset of trials (silent catch trials, 10\%), while the stimulus was normally played in the rest of the trials of the session (standard trials, 90\%) (Fig. 5a, middle and top). The RT distributions for standard trials were very similar to those observed in Group 1 (Fig. 5b; Supplementary Fig. 10, left column). Crucially, the distribution of proactive responses alone obtained from fitting the full PSIAM to RTs in standard trials predicted very accurately the distribution of RTs in silent trials (Fig. 5c; Supplementary Fig. 10 , right column). Removing the stimulus fully unveiled the RT distribution of proactive responses that was mixed with RTs from reactive responses in standard trials, providing evidence that within each trial the Al process evolves independently of the presentation of the stimulus.

The second basic hypothesis of the PSIAM is that proactive responses are triggered by a process time-locked to fixation onset, while reactive responses are triggered by a process locked to stimulus onset. The model predicts that varying the interval between fixation onset and stimulus onset should affect response timings by favoring one process over the other. In a second set of experiments, the stimulus was either advanced (advanced stimulus trials, $\Delta=-250$ or $-150 \mathrm{~ms}$ for Groups 2 or 3 , respectively; $5 \%$ of trials), or delayed (delayed stimulus trials, $\Delta=+50$ or $+150 \mathrm{~ms}$, Groups 2 or $3 ; 5 \%$ of trials) with respect to the standard condition $(\Delta=0$, standard trials, $90 \%$ ) (Fig. 5a, bottom). We first tested whether, in advanced and delayed stimulus trials, the RT distributions could be simply derived from the model fitted to standard trials but only shifting the onset of the EA process by the same amount $\Delta$. For delayed stimulus trials, the model with a shifted EA captured the RT distribution well: most responses were proactive, and the late stimulus onset caused a small bump of reactive responses at the tail of the RT distribution (Fig. 5e; Supplementary Figs. 11 and 12, right columns). This small impact of delayed stimulus was clearly visualized in time delay curves, which diverged from silent trials after an extra delay of $\Delta$ compared to standard trials, as expected (Fig. 5f). In contrast, for advanced stimulus trials, simply advancing the EA by $\Delta$ predicted faster responses than those observed in the rats (Fig. $5 d$, solid blue curve; Supplementary Figs. 11 and 12, left columns, solid blue curves). This suggests that, when 
stimulus is presented ahead of the expected time, the EA process takes longer to reach the decision bounds than in standard trials. Indeed, simply allowing the latency $t_{E}$ to be longer in these advanced trials provided a much better fit of the RT distribution (Fig. $5 \mathrm{~d}$, dashed blue curve; Supplementary Figs. 11 and 12, left columns, dashed blue curves). This additional delay, which increased with the magnitude of the advancement $\Delta$ (Fig. $5 \mathrm{~g}$ ), could reflect attentional delays related to the unexpected timing of the stimulus onset (Nobre, Correa and Coull, 2007; Vangkilde, Coull and Bundesen, 2012), or a signature of more complex interactions between $\mathrm{Al}$ and EA (see Discussion). Finally, adjusting the EA's drift $v_{E}$ instead of $t_{E}$ did not provide a better fit, and adjusting the two parameters together only caused a marginal average improvement (Fig. 5h; Supplementary Fig. 13). Overall, these experiments revealed that the PSIAM could account for how RT distributions are affected by changes in stimulus timing, and showed that EA latency is increased when stimulus timing is unexpected.

a
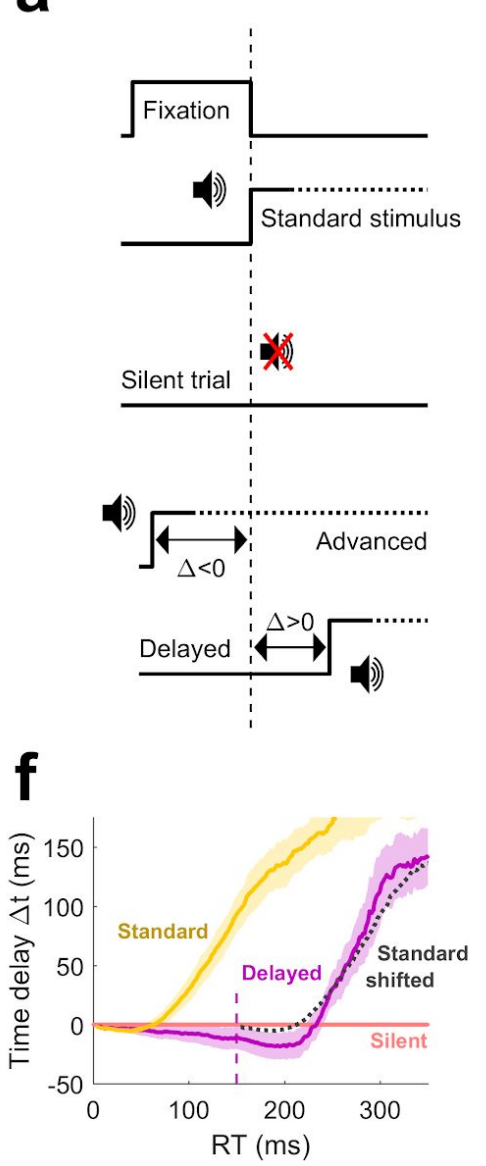

b

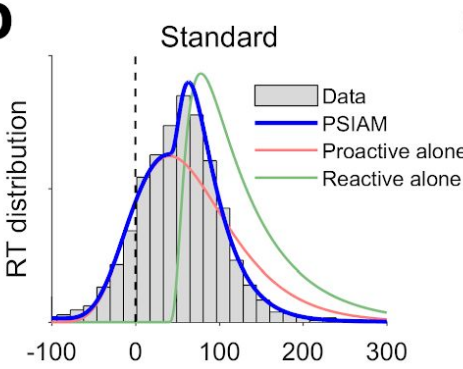

d

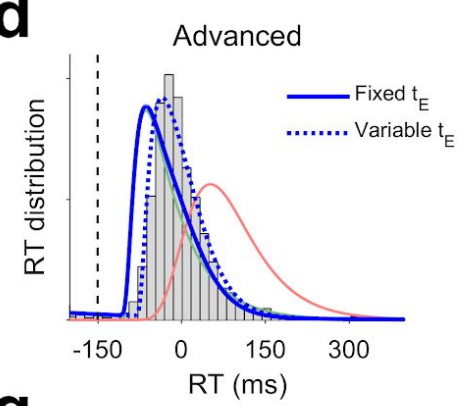

$\mathbf{g}_{6}$

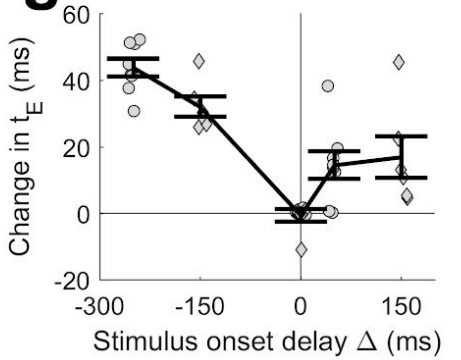

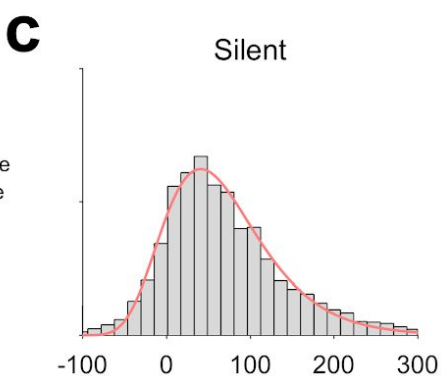

e

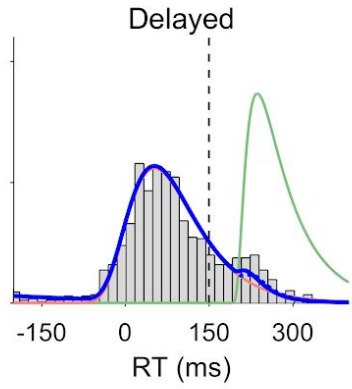

h

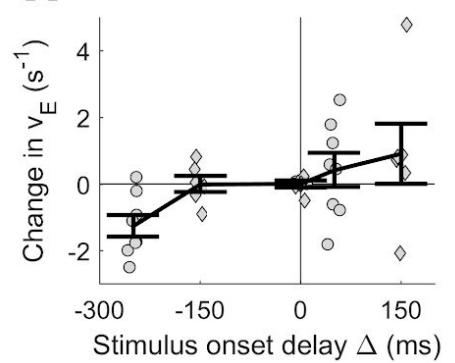

Fig. 5 | Reaction times in experiments with perturbed stimulus onset. a, In silent sessions, the stimulus was not presented in $10 \%$ of randomly selected catch trials (silent trial; Group 2, $n=6$ ). In advanced/delayed stimulus sessions, the stimulus was either advanced in $5 \%$ of trials $(\Delta=-150 \mathrm{~ms}$ for Group 2 and -250 ms for Group 3, n= 8) or delayed in $5 \%$ of trials $(\Delta=+150 \mathrm{~ms}$ in Group 2 and $+50 \mathrm{~ms}$ in Group 3) with respect to the onset time in standard trials. Responses were valid if they occurred after stimulus onset in advanced stimulus trials, and after fixation offset in silent and delayed stimulus trials. b, RT distribution for an example rat (gray bars; rat \#43) and model fit (blue line) for maximum stimulus strength $(s=1)$ in standard trials taken from sessions with silent catch trials. The distributions of proactive alone (red) and reactive alone (green) RTs generated by the Al and EA processes 
working in isolation are also shown. Vertical dashed line: stimulus onset. c, RT distribution in silent trials from the same rat shown in $b$ (gray bars). Model prediction corresponds to the RT distribution of proactive alone responses obtained from standard trials (red line; same as in $b$ ). $\mathbf{d}$, Same as in panel $b$ but for a different example rat in trials with advanced stimulus $(\Delta=-150 \mathrm{~ms}$; rat $\# 44)$. Model prediction corresponds to advancing the reactive alone density from standard trials by $150 \mathrm{~ms}$ (green line) while keeping the Al density (red line) unchanged (solid blue curve). Dashed blue curve shows model fit, where the EA parameter $t_{E}$ was fitted while keeping other EA and Al parameters unchanged. e, Same as in panel $d$ for delayed stimulus trials, but delaying the isolated EA density by $150 \mathrm{~ms}$ in the model prediction. $\mathbf{f}$, Time delay curves in standard and delayed $(\Delta=+150 \mathrm{~ms})$ trials for maximum stimulus strength $(s=1)$ versus silent trials (reference), averaged across rats. Dotted black line is the same as the standard condition, shifted $+150 \mathrm{~ms}$ for comparison. Vertical line marks the onset of the delayed stimulus. Shaded areas show s.e.m. g-h, Difference in the fitted values for $t_{E}(\mathrm{~g})$ and $v_{E}$ (f) between standard and advanced/delayed trials at each stimulus onset delay $\Delta$. Line: average across animals; error bars: s.e.m.; diamonds (Group 2) and circles (Group 3): individual rats.

\section{Slowing of the responses along the session is mediated by a decrease in Al speed}

The existence of the dual mechanism for response timing in the PSIAM suggests that the speed of response could be regulated by two different mechanisms: by a change in the decision bounds of the EA process, as classically reported (Bogacz et al., 2010); or by a modulation of the speed of the Al process, taking place independently of evidence accumulation. We assessed whether the systematic slowing of the RTs observed within each session in every animal (Fig. 6a) was mediated by the modulation of Al, at odds with the classical account of slowing based on decision bounds. Cumulative RT distributions sorted by trial index showed that there were fewer fixation breaks and fewer express responses at the end compared with the beginning of the session (Fig. 6b). In other words, the slowing already occured for responses triggered even before any reactive response could be performed (Fig. 6c). This suggested that the slowing was due at least in part to changes in the Al. We thus incorporated within-session slowing in the model by allowing the drift of the Al to decrease linearly with trial number within each session, while keeping the EA parameters constant across the session. Remarkably, this simple modification captured quantitatively the change in time delay curves observed within the session (Fig. 6d). The model reproduced the non-monotonicity of the curves with a dip around $130 \mathrm{~ms}$, indicating that the slowing in RTs was not as severe in that range of reaction times. In the PSIAM, this corresponded to the range where reactive responses, which remained unchanged throughout the sessions, dominated over proactive responses (Figs. $3 \mathrm{c}$ and $6 \mathrm{~d}$, top inset; Supplementary Fig. 8). Overall, the analysis suggests that the Al process, but not the EA, was affected by internal factors that changed consistently within each session, mediating a gradual slowing of responses. 

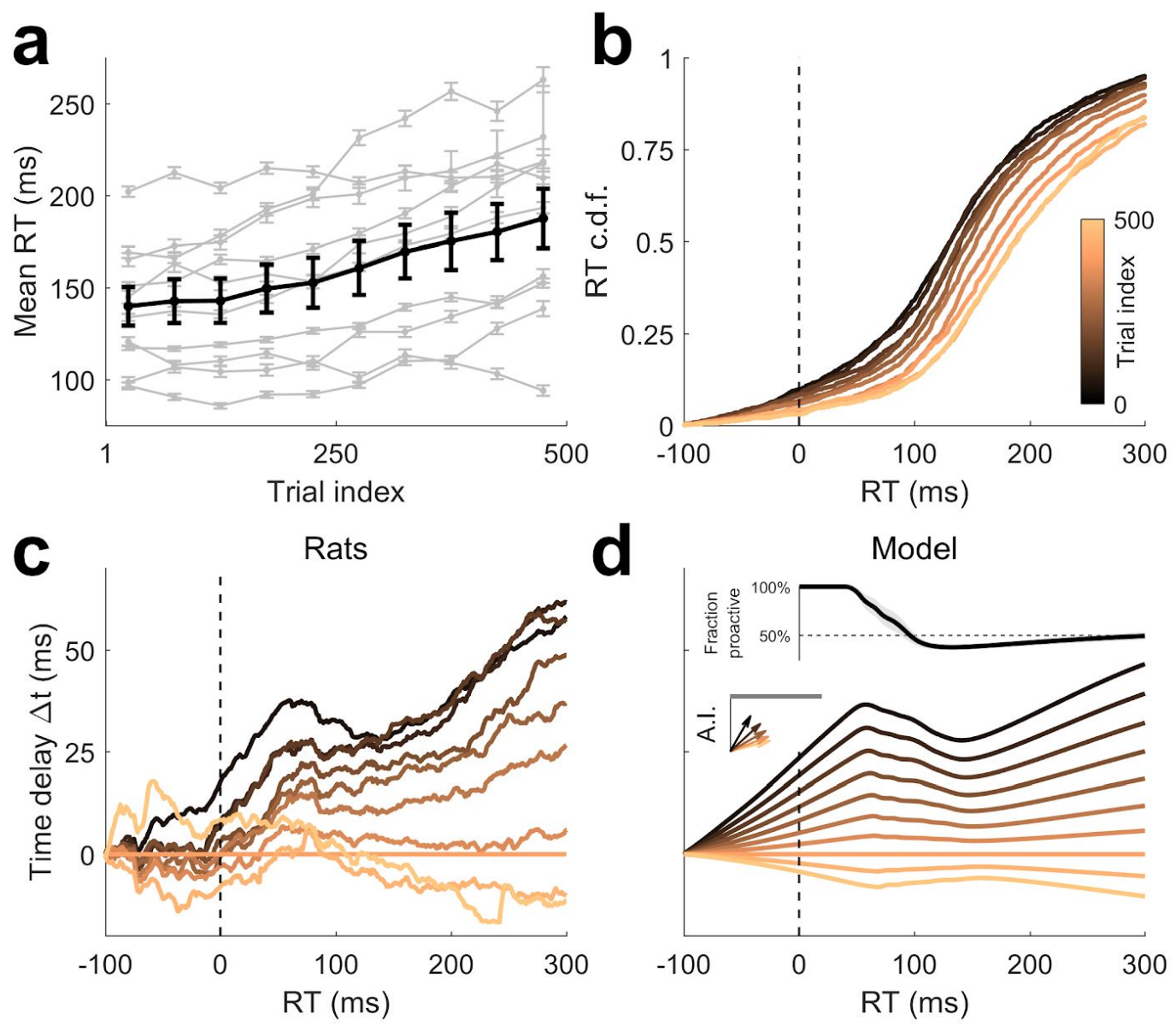

Fig. 6 | Within session slowing of responses. a, Mean RT as a function of trial position within the session. Thick line: group average, thin lines: individual rats (Group 1, $n=10)$. Trials were grouped in 50 trial blocks. Error bars: s.e.m. b, RT cumulative distribution for different trial blocks for an example animal (rat \#14). Color code in b-d indicates trial block. Vertical dashed line in b-d indicates stimulus onset. c, Time delay curves averaged across rats. Trial block 351-400 was taken as reference (i.e. corresponded to time delay $\Delta t=0)$. $\mathbf{d}$, Time delay curves simulated using the PSIAM fitted to the RTs for each animal, averaged across rats. Top inset: proportion of proactive vs reactive responses with RT as estimated by PSIAM for each animal, averaged across rats; shaded area: s.e.m. Left inset: sketch of the impact of trial index on Al's drift.

\section{Fatigue and satiety cause within session slowing by modulating Al speed}

The observed slowing of responses along the session could be linked to a change in two distinct internal variables of the animal (Logue and Peña-Correal, 1985; Richards et al., 1997; Simon et al., 2010; Reinagel, 2018) that increased as the session progressed: physical fatigue and satiety (Fig. $7 \mathrm{a}$, center and right). The number of completed trials and the amount of consumed reward since the beginning of the session provided proxies for fatigue and satiety, respectively. In order to disentangle the contribution of each of these factors, we performed additional experiments where the size of the reward was varied across sessions (Group 4, $n=4$; laterality discrimination task; see Methods). We first observed that the proportion of fast proactive responses $(R T<50 \mathrm{~ms})$ steadily decreased with trial index for all 
reward conditions, but the impact of reward size on RTs switched gradually along the session: while at the start of the session, a larger reward lead to a larger fraction of fast responses, the effect switched at the end of the session (Fig. 7b). To explain this, we incorporated fatigue and satiety in the model by allowing the drift of the Al process to change linearly with trial number and with the amount of consumed reward within the session (Fig. $7 \mathrm{a}$, top row). Moreover, since the reward size, fixed within a session, could also affect the rats' overall motivation and speed, we also let Al drift depend linearly with the reward size (Fig. 7a, left column). This extended model captured the impact of reward size on how the proportion of fast responses changed throughout the session (Fig. 7c). Trial index and consumed reward were found to have a negative impact on Al drift, i.e. slowed the Al process, whereas larger reward size had a positive impact, i.e. sped up the Al process. (Fig. $7 d$ ). Thus, the gradual switch of the reward size dependence along the session arose from the opposite contributions of reward size and reward consumption: while at the start of the session, animals in large reward sessions were faster because they were more motivated, at the end of the session the effect reversed because they were also more satiated. In sum, the analysis shows that the Al process was subject to systematic variations within and across sessions by means of changes in three factors: while larger reward sped up the Al process, fatigue and satiety slowed it down. 

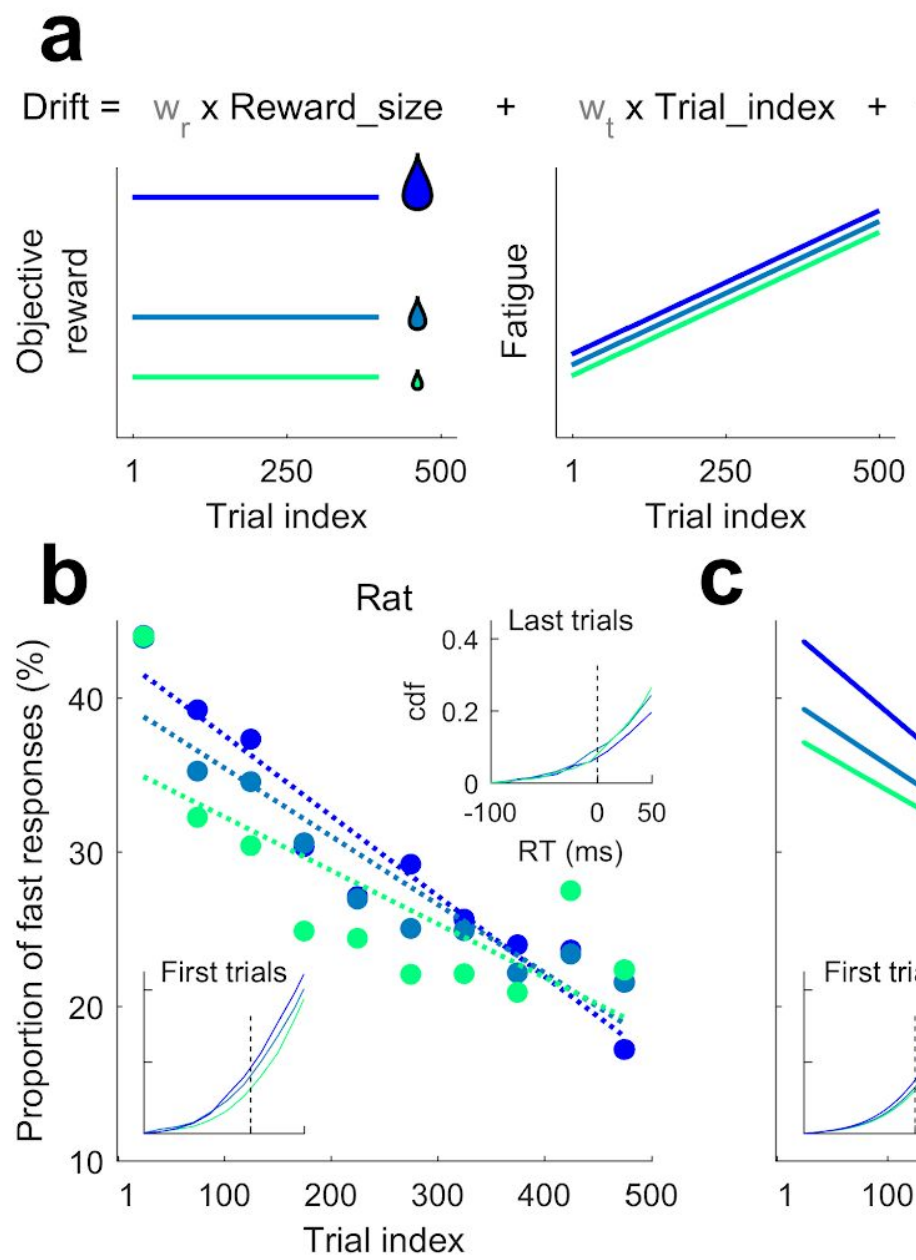

C
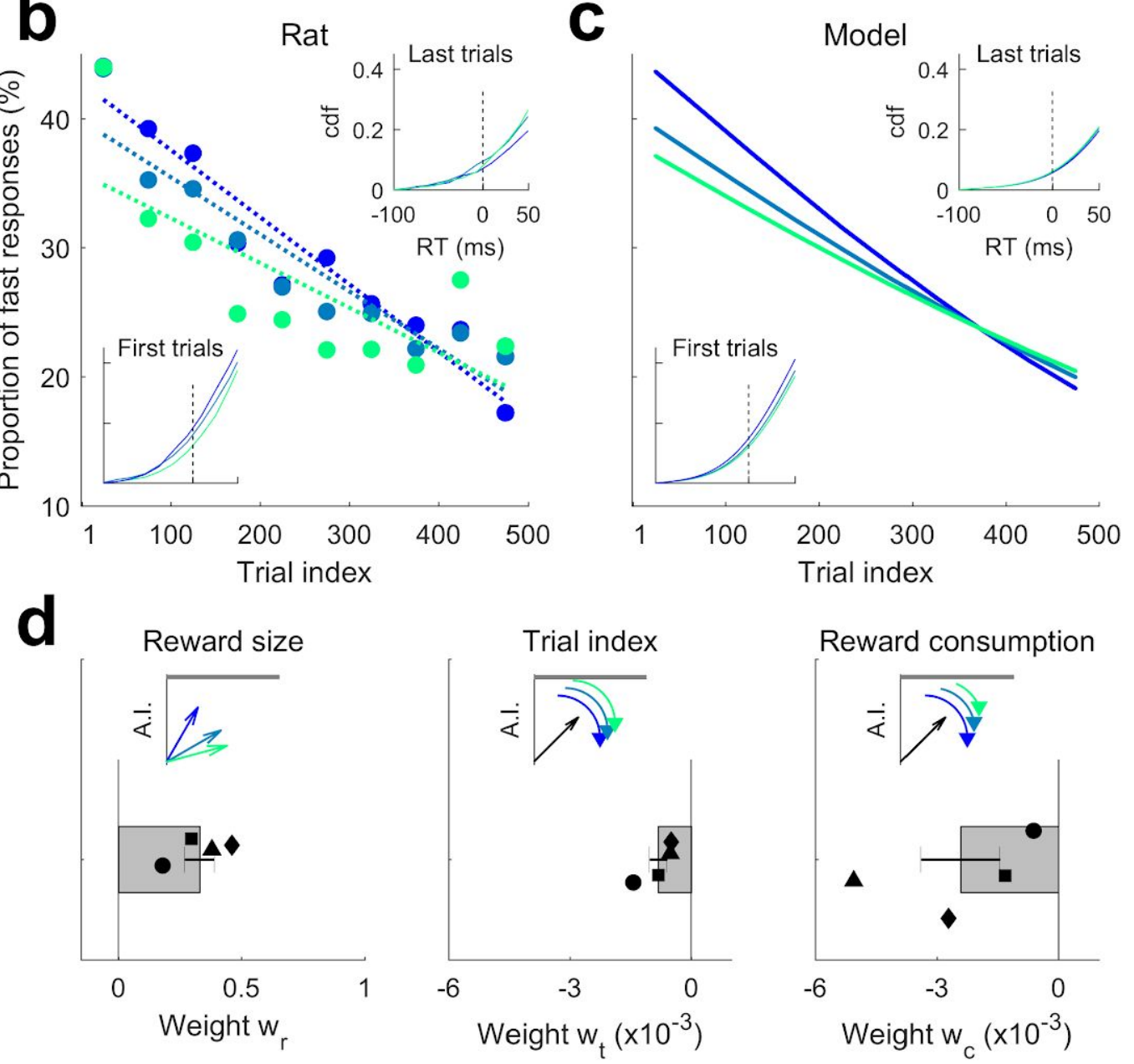

Fig. 7 | Internal factors impacting RTs. a, Reward size (left), fatigue (center) and satiety (right) as a function of trial position within session for low (green), middle (light blue) and high (strong blue) reward size sessions. Color code is shared for all panels a-d. Top equation illustrates the implementation in the model of these three features as linear regressors in Al drift. b. Proportion of rat's fast responses (faster than $50 \mathrm{~ms}$ after stimulus onset, or fixation break) as a function of trial position within session and reward size, for an example animal (rat \#54). Dashed lines: linear fits. Insets: RT cumulative distribution for trial block 51-100 (bottom left) and 451-500 (top right); vertical dashed line: stimulus onset. c, Proportion of fast responses simulated using PSIAM fit to the RTs of the example rat in b. 
Insets: same as b, but simulated using PSIAM. d, Estimated weight for reward size (left), trial index (center) and reward consumption (right) regressors in Al drift (Group 4). Bars: mean across animals; symbols: individual rats; error bars: s.e.m.

\section{DISCUSSION}

We used an acoustic discrimination task to characterize the mechanisms underlying the timing and choices of rats' perceptual decisions. We found that the pattern of responses could not be solely explained by traditional models of perceptual decision making whereby responses are triggered in reaction to the accumulated evidence crossing the bound (Carpenter and Williams, 1995; Drugowitsch et al., 2012; Verdonck and Tuerlinckx, 2016). In addition to such reactive responses, animals generated proactive responses in which the timing was stimulus-independent but the choice was stimulus-dependent (Fig. 2). A novel model, the PSIAM, proposes that each response type is triggered by one of two parallel processes: the Action Initiation, a proactive timing process that starts in anticipation of stimulus onset and ramps up until reaching a Go bound; and the Evidence Accumulation, a process initiated by the stimulus presentation that integrates sensory evidence until reaching one of the decision bounds (Fig. 3). The two processes run in parallel and race to induce a response, which is triggered as soon as one of the two reaches its respective bound. The Action Initiation process not only mediated premature responses but also generated slow responses, effectively implementing an urgency to respond when the EA process took too long to hit a decision bound (Fig. 4). As predicted by the model, proactive and reactive responses, which are locked respectively on the fixation onset and the stimulus onset, were dissociated in a second set of experiments where stimulus onset was perturbed (Fig. 5).

In psychophysics, it is common to find a small fraction of responses which are too fast to be triggered by the stimulus (Carpenter and Williams, 1995). They are usually considered as random anticipated responses and removed from subsequent analysis. Here we showed compelling evidence that, in our task, those express responses represented the lower tail of a wide distribution of proactive responses, whose timing was independent of the stimulus. For intermediate RTs, these proactive responses were present but they were mingled with reactive responses (Fig. 3c), as confirmed by experiments in which we omitted or shifted stimulus onset (Fig. 5). Three factors in our task could be promoting the anticipation of the Action Initiation process leading to a large fraction of proactive responses. First, the stimulus onset occurred at a fixed time interval after fixation onset (i.e. a fixed foreperiod), so that the rats could reliably time their withdrawal from the central port right after stimulus onset (Green, Smith and von Gierke, 1983). A random fixation period (Roitman and Shadlen, 2002) would probably lead to a more conservative strategy where the proactive process is slowed to avoid too early responses. Of note, temporal expectations also led to a reduction of the non-decision time of the EA process when the stimulus appeared at the predicted time in comparison to when it appeared earlier or later (Fig. $5 \mathrm{~g}$ ), in agreement with a recent observation (van den Brink et al., no date). Second, because of sensory delays, and because the response involves a full orienting body movement lasting 300-500 ms, the rat had time for finishing stimulus integration between leaving the central port (which interrupts the stimulus) and poking at one of the lateral ports. This makes express responses a viable 
strategy in which subjects leave the port right after stimulus onset, before stimulus can influence the motor plan, and integrate the sensory information while executing the orienting movement. The same type of online updating strategy could be applied for responses performed by arm movements, which can be swiftly adjusted en route to the target point (Resulaj et al., 2009); but perhaps not for more ballistic responses such as saccades or button presses. And third, the structure of the trial sequence allowed rats to partially predict the category of the upcoming stimulus based on previous trials' responses and rewards (Hermoso-Mendizabal et al., 2020). This partial predictability allowed animals to reach $\sim 65 \%$ accuracy for minimal stimulus durations in biased trials (Supplementary Fig. 3; note however that most of our analyses were performed on unbiased analyses, where rats did not use trial history to predict the next stimulus category). These features allow achieving both very fast and accurate responses, especially for high stimulus strength where short stimuli provide enough evidence to reach high levels of accuracy (Stanford et al., 2010) (Fig. 2d). In other words, animals are fooling the speed-accuracy trade-off (Smeets, Oostwoud Wijdenes and Brenner, 2016).

The Al process allows modulating reaction times not only based on the structure of the task, but also depending on motivational factors. We found that RTs slowed down within the session in all our animals (Fig. 6) (Davis, Roma and Hienz, 2016). Previous literature has investigated whether the impact of session time on RTs reflects the accumulated fatigue (physical and/or cognitive) or the increase of satiety. One study found that, in a delay discounting task, the increase in uncompleted trials observed during the session was larger for aged rats compared to young animals (Simon et al., 2010). Since food consumption did not differ between the two age groups, the authors argued that the main driving factor was fatigue rather than satiation. In humans too, responses slow down when the subject is fatigued (Welford, 1988; Faber, Maurits and Lorist, 2012). On the other hand, other studies have found that food or water satiety increases the latency of responses in rats and pigeons (Logue and Peña-Correal, 1985; Richards et al., 1997). We disentangled the effect of fatigue and satiety by varying the reward size across sessions in new experiments, and found that both factors contributed to within-session slowing (Fig. 7). Furthermore, our model-based approach allowed us to identify the cognitive mechanisms underlying this effect: both fatigue and satiety impact the drift of the Al process, i.e. slow down the initiation of proactive responses. On the contrary, increasing reward size led to higher Al drift, and thus faster responses. This shows that internal states can modulate decision-making through the Al, without modulating Evidence Accumulation.

The idea that the timing of perceptual decisions depends on an internal sense of time pressure is not novel (Churchland, Kiani and Shadlen, 2008; Bowman, Kording and Gottfried, 2012; Drugowitsch et al., 2012; Thura et al., 2012; Hanks, Kiani and Shadlen, 2014; Purcell and Kiani, 2016). Several previous studies have proposed the existence of an urgency signal that interacts with the integration of sensory evidence in order to avoid slow responses. A popular idea is to incorporate urgency directly into the DDM, while keeping decision-making as a serial process where action initiation follows evidence integration. This can be done by gradually collapsing the decision bounds (Bowman, Kording and Gottfried, 2012; Drugowitsch et al., 2012), or by a ramping evidence-independent signal that either increases the gain of evidence accumulation (Thura et al., 2012) or adds a non-specific input 
to all possible response activations, promoting motor actions (Churchland, Kiani and Shadlen, 2008; Hanks, Kiani and Shadlen, 2014; Purcell and Kiani, 2016). All these mechanisms account for the fact that very slow responses are usually less frequent in participants than what the DDM with fixed bounds would predict. However, in all these extensions of the DDM, RTs always depend on the evidence accumulation since all responses are triggered after reaching a decision bound, so these models cannot explain express responses, where RT is independent of stimulus strength whereas choice is contingent on stimuli (35\% of all responses in our dataset, SD=25\%; Fig. 2). By contrast, the PSIAM provides an alternative approach where a single mechanism, i.e. proactive responses, can account for (1) express responses yielding stimulus-independent RTs and stimulus-dependent choices (Fig. 3d-e), and (2) the low probability of very slow responses found in the same dataset (Fig. 4a). In the PSIAM, Al sets a stimulus-independent internal deadline for triggering the response, avoiding long accumulation-to-bound processes in EA. As such, the action of the Al can be viewed as provoking the instantaneous collapse of decision bounds on the EA at a stochastic time defined by the Al bound crossing. While in our task the Al most often reached the Go bound shortly after stimulus onset, under other circumstances, a slower Al drift could be used to trigger proactive responses after a longer stimulus duration. Indeed, a slower and more precise Al process can shift the timing of proactive responses, from early to late responses, i.e. from causing express responses to ensuring an upper bound on integration time (Fig. 8a). Such a change also leads to a larger drop in accuracy associated with long RTs (Fig. 8b), an effect that is commonly observed in the decay of tachometric curves (Murphy, Boonstra and Nieuwenhuis, 2016; Devine et al., 2019), but is usually attributed to either the variability in the drift of the evidence accumulation process or the gradual collapse of the decision bounds. Adjusting $\mathrm{Al}$ parameters in the PSIAM provided a more flexible way to set the speed-accuracy trade-off compared to modulating the decision bounds of the EA process (Milosavljevic et al., no date; Reddi and Carpenter, 2000). Hence, while rats may lay on the impatient side of Al, Al in humans and non-humans primates performing classical perceptual tasks may primarily be used to prevent very long reactive responses (Murphy, Boonstra and Nieuwenhuis, 2016). 

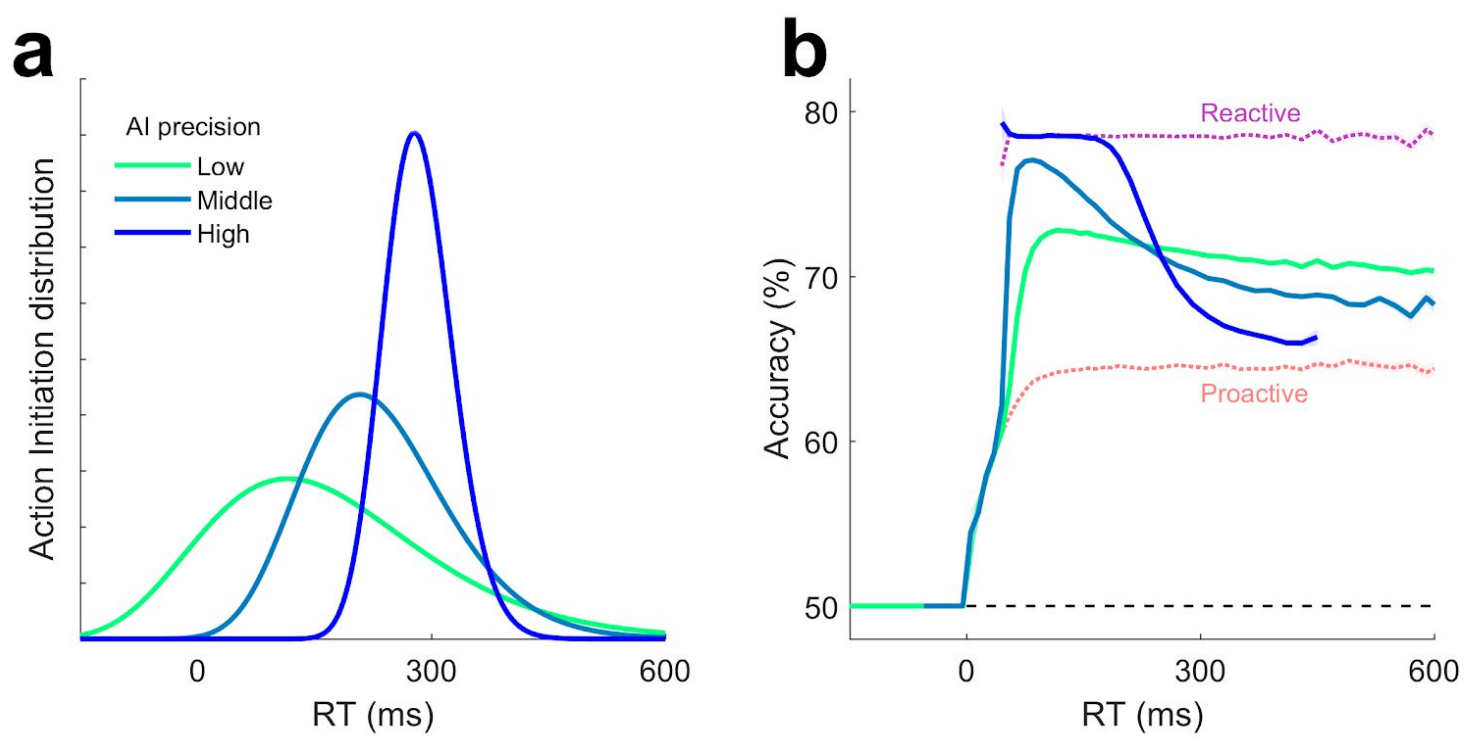

Fig. 8 | Modulation of accuracy with manipulations of Al. a, Distribution of proactive alone RTs for three simulated manipulations varying the latency and the degree of temporal precision of the Al. $\mathbf{b}$, Simulated tachometric curves obtained from the PSIAM using the Al processes shown in a and stimuli of intermediate strength $(s=0.5)$. Lapse responses were not incorporated into the model. We also simulated tachometric curves for the proactive (pink) and reactive (purple) responses; these two tachometric curves are independent of Al manipulations, and provide lower and upper bounds on accuracy, respectively.

The PSIAM is also related to two previous models of sensory guided responses with a proactive component. In one study, human subjects were forced to respond "earlier-than-normal" in a visually guided reaching task (Haith, Pakpoor and Krakauer, 2016). A simple model separating the when and what decisions into two separate processes could elegantly explain RTs and choices: the Action Initiation process always determined the timing of response, whereas a second stimulus-dependent Action Preparation process determined the choice but never the timing (Haith, Pakpoor and Krakauer, 2016; Hardwick et al., 2019). While this model, like the PSIAM, can generate proactive responses where the choice is informed by the Evidence Accumulation process, it cannot explain that some reaction times depend on stimulus evidence, a clear signature of reactive responses present in our data (Figs. 3 and 5) and in many other studies (Roitman and Shadlen, 2002; Pardo-Vazquez et al., 2019). An alternative model, based on data from monkeys performing an oculomotor visually-guided task under strong external time pressure, proposes a race between choice-selective Action Initiation processes which are accelerated or decelerated by the presence of a stimulus (Salinas et al., 2010; Stanford et al., 2010; Shankar et al., 2011). By combining proactive and reactive processes into a single "stimulus-dependent race-to-bound" process, the model can capture the existence, like in our data, of two underlying components of the RT distribution: responses that are time locked to the onset of the foreperiod (the fixation onset in our task) and responses that are time-locked to the stimulus onset (Stanford et al., 2010). The PSIAM differs however from this model in two important aspects. First, the PSIAM incorporates the classical mechanisms of Evidence Accumulation, which captures the dependency of RTs on stimulus strength in standard free response paradigms, something not present in the "accelerated race" model of (Stanford et 
al., 2010). Second, the PSIAM grounds on separate accumulation-to-bound EA and Al processes, whereas the accelerated race model features proactive and reactive mechanisms in a single accumulation-to-bound process. A single accumulation-to-bound process is incompatible with responses where the stimulus impacts choice but not RT, such as express responses in our rats. Thus, our data support that reactive responses are mediated by a separate accumulation-to-bound process independent from the proactive Al process. A direct extension of the PSIAM, inspired by the accelerated race model, is to assume that EA does not trigger responses when reaching bound but rather accelerates the ongoing Al process towards the Go bound (Stanford et al., 2010). This extension of PSIAM would reconcile the two models, and could account, for the increased EA latency found in advanced trials (Fig. 5d,g).

Activity in multiple brain areas shows correlates of processes like the Al or EA of the PSIAM. Neurons showing slow ramping activity preceding proactive responses have been found in several brain areas such as the cortical supplementary motor area (Romo and Schultz, 1992), the frontal eye field (Stanford et al., 2010), the secondary motor cortex (Murakami et al., 2014), the lateral intraparietal area (Maimon and Assad, 2006) and the basal ganglia (Lee and Assad, 2003). Interestingly, some neurons showed ramping activity specifically before proactive but not reactive responses (Romo and Schultz, 1992; Maimon and Assad, 2006). Similarly, a large number of cortical and subcortical areas has been suggested to encode accumulated stimulus evidence (Kim and Shadlen, 1999; Roitman and Shadlen, 2002; Gold and Shadlen, 2007; Ding and Gold, 2013; Thura and Cisek, 2014; Hanks et al., 2015; Yartsev et al., 2018). There is conflicting evidence regarding whether neural markers of accumulated evidence are influenced by the level urgency (Churchland, Kiani and Shadlen, 2008; Park et al., 2014; Thura and Cisek, 2014; Steinemann, O'Connell and Kelly, 2018). Thus, despite being a phenomenological model aimed to describe behavioral responses, by postulating the existence of race dynamics between proactive and reactive processes, the PSIAM model may become an instrumental tool to identify the underlying mechanisms of neural ramping dynamics during perceptual decisions.

In conclusion, our analyses support a model where decision-making is mediated by two separate processes, Action Initiation and Evidence Accumulation, which race to trigger a behavioral response. They also point to the Al process as a flexible mechanism allowing strategic and idiosyncratic adjustments of the action timing to multiple factors, such as temporal and choice predictability, motivation and urgency.

\section{METHODS}

All experimental procedures were approved by the local ethics committee (Comité d'Experimentació Animal, Universitat de Barcelona, Spain, Ref 390/14).

\section{Animal Subjects}


Animals were male Long-Evans rats ( $n=26,350-650 \mathrm{~g}$; Charles River), pair-housed during behavioral training and kept on stable conditions of temperature $\left(23^{\circ} \mathrm{C}\right)$ and humidity $(60 \%)$ with a constant light-dark cycle (12h:12h, experiments were conducted during the light phase). Rats had free access to food, but water was restricted to behavioral sessions. Free access to water was provided on days with no experimental sessions.

\section{Task description}

Rats in Group 1 ( $n=10)$ performed an auditory reaction-time two-alternative forced choice task. Specifically, this group of rats performed a frequency discrimination version of the task (Hermoso-Mendizabal et al., 2020). Briefly, at each trial, an LED on the center port indicated that the rat could start the trial by poking in (Fig. 1). After a fixation period of $300 \mathrm{~ms}$, the LED switched off and an acoustic stimulus consisting in a superposition of two amplitude-modulated frequencies was presented (see details below). Each frequency was associated with a specific side and reward was available at one of the two lateral ports, depending on the dominant frequency. Animals could respond any time after stimulus onset. Correct responses were rewarded with a $24 \mu \mathrm{L}$ drop of water and incorrect responses were punished with a bright light and a $5 \mathrm{~s}$ time-out. Trials in which the rat did not make a side poke response within 4 seconds after leaving the center port were considered invalid trials and were excluded from the analysis (average of $0.4 \%$ invalid trials per animal). Withdrawal from the center port before stimulus onset cancelled stimulus presentation (Fixation Break, FB). After a FB, rats were allowed to initiate fixation again, with no time-out, and as many times as necessary until fixation was complete (indicated by center LED offset). Rats performed an average of 694 trials per session (range: 335 -1188), one session per day lasting 60-90 min, 6 days per week, during 9 months. The data presented in this study was taken from the period after training yielding an average of 56,506 valid trials per rat.

Rats in Group $2(n=6)$, Group $3(n=8)$ and Group $4(n=4)$ performed an intensity level discrimination version of the task (Pardo-Vazquez et al., 2019; Hermoso-Mendizabal et al., 2020). In this task, two speakers positioned at both sides of the box played simultaneously an amplitude-modulated white noise. Rats had to discriminate the side with the loudest sound (right or left) and seek reward in the associated port. The details of the task are exactly the same as in the previous one, but in these groups the time-out after error responses was of $2 \mathrm{~s}$ and was not associated with bright light. Rats in Group 2 performed sessions with silent catch trials (random 10\% of trials without sounds; range: $45-50$ consecutive sessions) in which they could respond any time after the $300 \mathrm{~ms}$ fixation offset, as in standard trials. Rats in Groups 2 and 3 performed sessions with advanced/delayed stimulus catch trials (random $10 \%$ of trials varying sound onset; $45-50$ consecutive sessions). For $5 \%$ randomly selected trials, the stimulus onset time was advanced $\Delta=-150$ ms for animals in Group 2 and $\Delta=-250 \mathrm{~ms}$ for rats in Group 3 (Fig. 5a, advanced stimulus trials); and for another $5 \%$ of trials, it was delayed $\Delta=+150 \mathrm{~ms}$ for Group 2 and $+50 \mathrm{~ms}$ for Group 3 (Fig. 5a, delayed stimulus trials). For these advanced/delayed catch trials, stimulus strength was always set to maximum $(s=1)$ to gain statistical power, and to enhance evidence-triggered, reactive responses. In advanced stimulus and standard trials, rats could respond any time after fixation offset (either at 150 or at $50 \mathrm{~ms}$, and at $300 \mathrm{~ms}$, respectively), 
which coincided with stimulus onset. In delayed stimulus trials, rats could respond any time after fixation offset (300 ms), which was dissociated of stimulus onset (either at 450 or at 350 $\mathrm{ms}$ ). For rats in Group 2, which participated in silent and advanced/delayed stimulus sessions, the order of the sessions was balanced across animals, with 3 rats starting with the advanced/delayed catch trials sessions and 3 starting in the silent catch trials sessions. The overall fraction of catch trials was maintained low at $10 \%$ to avoid animals modifying substantially their original behavior during these sessions.

Rats in Group 4 performed sessions with a modified water reward size. In small-reward sessions, the amount of water obtained in each correct response $(12 \mu \mathrm{l})$ was half of the reward in standard sessions. In large reward size sessions, the amount of water obtained $(48 \mu \mathrm{l})$ was double the amount in standard sessions (Fig. 7a, left). The three types of sessions were randomly interleaved.

For Group 1, the behavioral setup (Island Motion, NY) was controlled by a custom software developed in Matlab (Mathworks, Natick, MA), based on the open-source BControl framework (http://brodylab.princeton.edu/bcontrol). For Groups 2-4, the behavioral setup was controlled by BPod electronic boards (by Sanworks) and the task was run using the Python-based open software package PyBPod (http://pybpod.com/).

Additional details on the acoustic stimulus and the stimulus sequence are provided in the Supplementary Information Methods.

\section{Reaction times}

Reaction time (RT) was defined as the time elapsed from stimulus onset to center port withdrawal (Fig. 1b). For standard trials, RT matched the stimulus duration, as stimulus was switched off at center port withdrawal. For silent, advanced stimulus and delayed stimulus trials, RT was measured with respect to standard stimulus onset. Fixation breaks (FBs; $16 \pm 5$ $\%$ of total withdrawals, mean \pm standard deviation) were defined as withdrawals from the center port during the fixation period (300 ms for standard, silent and delayed stimulus trials; either 150 or $50 \mathrm{~ms}$ for advanced stimulus trials). Only the first center port withdrawals of each trial, either FB or RT, were analyzed: after a FB, further FBs and the subsequent valid response were discarded to remove possible serial effects within a single trial. Trials with RTs longer than $1 \mathrm{~s}$ were removed from the analysis $(0.5 \pm 0.6 \%$ of total withdrawals).

\section{RT-evidence Modulation onset}

The onset times for the modulation of the RT distributions by the stimulus strength (Fig. 2a-b) were computed similarly as in Supplementary Fig. 2b of (Pardo-Vazquez et al., 2019). For each time $t$, we computed a one-tailed Kolmogorov-Smirnoff test comparing the RT cumulative distributions for trials with strongest and weakest stimulus evidence (stimulus strength $s=1$ versus stimulus strength $s=0$ ), including both biased and unbiased trials, and 
excluding all reaction times larger than $t$. For each rat, we defined the modulation onset as the minimal value of $t$ at which this comparison became significant $(p<0.05)$.

\section{Time delay curves}

Time delay curves assess how much faster or slower is a given stochastic time variable with respect to a reference distribution; see Supplementary Fig. 3 of (Pardo-Vazquez et al., 2019). Intuitively, it represents the horizontal distance at any time point between a given cumulative distribution and a reference cumulative distribution (Supplementary Fig. 2). At a given time point $T$, time delay value is defined as the time difference $T r-T$, where $T_{r}$ is the time at which the value of the reference cumulative matches the value of the cumulative of interest at $T$, i.e. $C(T)=C_{r}\left(T_{r}\right)$. Positive time delay means that, at time $T$, there is a larger proportion of RTs lower or equal to $T$ in the condition of interest than in the reference condition.

\section{Parallel Sensory Integration and Action Model (PSIAM)}

In order to characterize the patterns of rat RTs and choices, we built the Parallel Sensory Integration and Action model (PSIAM) by combining a standard DDM with constant bounds (Ratcliff, 1978) (i.e. Evidence Accumulation process or EA process) with a second drift diffusion process modeling the timing of proactive responses (Action Initiation process or $\mathrm{Al}$ process; Fig. 3a-b, bottom red traces). EA is a Wiener process which integrates the evidence provided by the stimulus over time, following:

$$
\begin{aligned}
& \frac{d x(t)}{d t}=V_{E}+\sigma_{E} \cdot \xi(t), \quad \text { for } x \in\left(-\theta_{E},+\theta_{E}\right) \\
& \frac{d x(t)}{d t}=0, \quad \text { for } x= \pm \theta_{E}
\end{aligned}
$$

where $x(t)$ is the EA process representing the instantaneous accumulated evidence. The parameter $V_{E}$ is the drift which only depends on the stimulus evidence (see below); $\sigma_{E}$ is the noise variance, set to 1 to make the model identifiable; and $\xi(t)$ is a white noise stochastic process which represents the stimulus temporal fluctuations as well as the internal noise associated with the accumulation process. The EA process is initiated at value $x=z_{E}$ (starting offset) with a delay of $t_{E}^{\text {aff }}$ with respect to stimulus onset $t_{\text {stim }}$ (afferent latency); $x=$ $\pm \theta_{E}$ are the two symmetric decision bounds (Fig. 3a-b, and see below).

Action Initiation represents the preparation of a commitment to take a decision; it is modeled as an independent, one-bound DDM (Wald, 1947):

$$
\begin{array}{ll}
\frac{d y(t)}{d t}=V_{A}+\sigma_{A} \zeta(t), & \text { for } y<\theta_{A} \\
\frac{d y(t)}{d t}=0, & \text { for } y=\theta_{A}
\end{array}
$$

where the parameter $V_{A}$ is the drift of the $\mathrm{Al} ; \sigma_{\mathrm{A}}$ is the noise variance (always set to 1 ); and $\zeta(t)$ is a white noise stochastic process representing the internal variability associated with 
the precise timing of the Go action. The Al process is initiated at fixation onset with a delay of $t_{A}^{\text {aff }}$ (afferent latency); and $\theta_{A}$ is the action bound (Fig. 3a-b).

The PSIAM model distinguishes two types of responses, proactive and reactive, depending on the outcome of the "race" between the Al and the EA processes, i.e. depending on which process reaches the bound first. Reactive responses are generated when either of the two EA boundaries is reached first; the choice is then defined by which bound is hit (by convention, the upper bound corresponds to the rightwards choice, and the lower bound to the leftwards choice). Proactive responses are generated when the Al threshold is reached first; the choice is then defined as a direct read-out of the sign of the EA process $x(t)$ after the interrupted stimulus is integrated. This is equivalent to an instantaneous collapse of both EA's decision thresholds, i.e. a vertical bound in the EA (Fig. 3a). The RT at each trial is thus set by the first of the two processes to reach the bound, and corresponds to the time elapsed from stimulus onset to first bound hit, with an additional efferent delay $t_{\text {motor }}$ representing motor latency. The probability density function (pdf) $p(t)$ and the cumulative distribution function (cdf) $c(t)$ of the RTs depend on the pdf and cdf of the RTs that would be generated by either the $\mathrm{Al}\left(p_{A}(t)\right.$ and $\left.c_{A}(t)\right)$ or by the $\mathrm{EA}\left(p_{E}(t)\right.$ and $\left.c_{E}(t)\right)$ in isolation, through (Fisher and Tippett, 1928):

$$
\begin{aligned}
& p=p_{A} \cdot\left(1-c_{E}\right)+p_{E} \cdot\left(1-c_{A}\right) \\
& c=c_{A}+c_{E}-c_{A} \cdot c_{E}
\end{aligned}
$$

where we have dropped the dependence on $t$ to ease the notation. The distribution of the RTs generated in isolation by the Al is an Inverse Gaussian (IG) distribution (a.k.a. Wald distribution), so that the pdf and cdf for Al (Tweedie, 1945; Wald, 1947) are:

$$
\begin{aligned}
& p_{A}\left(t \mid V_{A}, \theta_{A}, t_{A}\right)=\theta_{A} \cdot \frac{1}{\sqrt{2 \pi \cdot\left(t-t_{A}\right)^{3}}} \cdot \exp \left(-\frac{V_{A}^{2}}{2} \cdot \frac{\left[\left(t-t_{A}\right)-\theta_{A} / V_{A}\right]^{2}}{\left(t-t_{A}\right)}\right) \\
& c_{A}\left(t \mid V_{A}, \theta_{A}, t_{A}\right)=\Phi\left(V_{A} \cdot \frac{\left(t-t_{A}\right)-\theta_{A} / V_{A}}{\sqrt{t-t_{A}}}\right)+\exp \left(-2 \cdot V_{A} \cdot \theta_{A}\right) \cdot \Phi\left(-V_{A} \cdot \frac{\left(t-t_{A}\right)+\theta_{A} / V_{A}}{\sqrt{t-t_{A}}}\right)
\end{aligned}
$$

where the parameter $V_{A}$ is the reduced drift $\left(\sigma_{A}=1\right)$ of the $\mathrm{Al} ; \theta_{A}$ is the reduced single-threshold height from the starting value of the Al process; and $t_{A}=t_{A}{ }^{\text {aff }}+t_{\text {motor }}$ is the total Al latency, summing afferent and motor latencies. The independent RT pdf for the EA alone corresponds to that of the standard DDM with two constant bounds (Wald, 1947; Samuels and Feller, 1969):

$$
\begin{aligned}
& p_{E}\left(t \mid V_{E}, \theta_{E}, Z_{E}, t_{E}\right)=p_{E}{ }^{+}+p_{E}^{-}=p_{E}^{-}\left(t \mid-V_{E}, \theta_{E},-Z_{E}, t_{E}\right)+p_{E}{ }^{-}\left(t \mid V_{E}, \theta_{E}, Z_{E}, t_{E}\right) \\
& p_{E}{ }^{-}\left(t \mid V_{E}, \theta_{E}, Z_{E}, t_{E}\right)= \\
& =\frac{\pi}{\left(2 \theta_{E}\right)^{2}} \cdot \exp \left(-V_{E} \cdot\left(Z_{E}+\theta_{E}\right)-\frac{V_{E}{ }^{2}}{2} \cdot\left(t-t_{E}\right)\right) \cdot \sum_{k=1}^{\infty}\left[\sin \left\{k \cdot \pi \cdot \frac{Z_{E}+\theta_{E}}{2 \theta_{E}}\right\} \cdot \exp \left(-\frac{k^{2} \pi^{2}}{2 \cdot\left(2 \theta_{E}\right)^{2}} \cdot\left(t-t_{E}\right)\right)\right]
\end{aligned}
$$

where $p_{E}^{ \pm}$are the joint pdfs for RT and choice, with + and - for choice corresponding to upper bound and lower bound, respectively. The parameter $V_{E}$ represents the reduced drift 
$\left(\sigma_{E}=1\right) ; \pm \theta_{E}$ represent the reduced upper and lower threshold values; $Z_{E}$ represents the reduced starting offset or bias; and $t_{E}=t_{E}^{a f f}+t_{\text {motor }}$ represents the total response latency time for the EA, summing afferent and motor latencies. The corresponding cumulative distribution $c_{E}$ is obtained by integrating Eq. 7 analytically over $t$.

\section{Model fit to reaction times data}

The fit of the PSIAM to the experimental RTs data was performed under Maximum Likelihood Estimation (MLE), using simplex method (Matlab function fmincon) with 20 random initial points in bounded parameter space to avoid local minima. Model parameters were estimated for each animal only based on RTs for all trials including FBs. The value of the EA starting point, EA drift and Al drift depended on trial conditions. EA drift $V_{E}$ scaled linearly with signed stimulus strength $S$ (positive/negative sign for rightward/leftward evidence), and we fitted the proportionality parameter $v_{E}$, i.e. $V_{E}=v_{E} \cdot S$. For a given trial $k$, the signed stimulus strength $S_{k}$ was computed as the instantaneous evidence $S_{k, f}$ averaged across frames presented in this trial, i.e. $\left\langle S_{k, f}\right\rangle_{f}$. To account for the slowing of the responses within each session (Fig. 6a-b), Al drift scaled linearly with trial index $k$ as $V_{A, k}=$ $v_{A O}+v_{\text {trial }} k$, and we fitted the parameters $v_{A O}$ and $v_{\text {trial }}$. EA initial offset $Z_{E}$ captured the animal's expectation of which response will be rewarded in the next trial. For unbiased trials, this offset was set to 0, i.e. no expectation. For biased trials (Supplementary Figs. 3 and 5), the value of the starting point was signed depending on the trial-dependent expectation of rewarded side $b_{k}= \pm 1$ (see Supplementary Methods), so that $Z_{E}=z_{E} \cdot b_{k}$, where we fitted the parameter $z_{E}$ representing the magnitude of the animal's expectation. The rest of the parameters of the PSIAM were constant for each animal. Additionally, we included a Huber's $\varepsilon$-contamination model for MLE robustness (Huber, 1964). Contaminant responses were included to avoid estimation parameters to be biased by very early FBs (especially numerous after error trials) and very late RTs (Ratcliff and Tuerlinckx, 2002). Contaminant responses were modelled from the mixture of a decaying exponential distribution and a uniform distribution as:

$$
\begin{aligned}
& p=c \cdot p_{C}+(1-c) \cdot p_{\text {model }} \\
& p_{C}(t \mid d, \beta)=d \cdot \beta \cdot \exp (-\beta \cdot t)+(1-d) \cdot c t t
\end{aligned}
$$

where $p, p_{C}$ and $p_{\text {model }}$ are the final, contaminant, and PSIAM densities respectively; the parameter $c$ is the proportion of contaminant responses; $d$ is the exponential-uniform mixture parameter; $\beta$ is the inverse of the exponential time constant; and ctt is the normalization constant for the uniform density alone. The PSIAM with contaminants has a total of 11 parameters: 4 for the Al (drift intercept $v_{A O}$ and trial-index weight $v_{\text {trial }}$, threshold $\theta_{A}$, and response latency time $t_{A}$ ); 4 for the EA (drift stimulus weight $v_{E}$, threshold $\theta_{E}$, response latency time $t_{E}$, and starting offset $z_{E}$ ); and 3 parameters for the contaminant distribution (proportion of contaminant responses $c$, the exponential-uniform mixture parameter $d$, and the inverse of the exponential time constant $\beta$ ). We added lower bound and upper bounds in the parameter search, both to speed up the optimisation algorithm and to confine the search within a range of a priori defined values. The bounds in parameter space set for MLE were: 
$[0,12] \mathrm{s}^{-1}$ for $v_{A 0} ;[-2,1] \cdot 10^{-2} \mathrm{~s}^{-1}$.trial ${ }^{-1}$ for $v_{\text {trial }} ;[0.1,10]$ for $\theta_{A} ;[-600,300] \mathrm{ms}$ for $t_{A} ;[2,10] \mathrm{s}^{-1}$ for $v_{E} ;[0.1,1.2]$ for $\theta_{E} ;[35,75] \mathrm{ms}$ for $t_{E} ;[-1 / 3,1 / 3]$ for $z_{E} ;[0,0.5]$ for $c ;[0,1]$ for $d$; and $[0,50] \mathrm{s}^{-1}$ for $\beta$. There was one less parameter to fit for unbiased trials as we set $z_{E}$ to 0 . The infinite series in the EA independent RT pdf (Eq. 8) and cdf were approximated numerically (Navarro and Fuss, 2009; Blurton, Kesselmeier and Gondan, 2012; Gondan, Blurton and Kesselmeier, 2014).

\section{Model prediction for choice data}

The PSIAM choice (Right/Left) corresponds to the sign of the final value of the EA process (Fig. 3a-b). For each rat, we computed the choice prediction (Figs. 3e and 4d) by simulating the PSIAM (Eqs. 1-2) using MLE parameter values. It should be stressed that parameter MLE values were obtained using RTs data only, and not choices. We also included some trial-to-trial variability in the EA starting offset (otherwise choices for very short reaction times would always be determined by the side of $Z_{E}$, i.e. the expectation, unlike what is observed in rats). The distribution of starting point was thus taken as a Beta distribution (stretched to cover the full domain of the EA process $\left.\left[-\theta_{E}, \theta_{E}\right]\right)$. The parameters of the distribution were set such that it had mean $z_{E}$ and standard deviation equal to $10 \%$ of the interbound distance between bounds (i.e. $0.2 \cdot \theta_{E}$ ). Simulations were run by discretizing differential equations (Eqs. 1-2) using Euler method with a time step of $0.1 \mathrm{~ms}$. We performed a total of $10^{7}$ trial simulations per rat.

\section{Model prediction for reaction times in catch trials with manipulated stimuli}

The density $p_{\text {silent }}$ of RTs in silent catch trials was predicted from the PSIAM using parameters fitted to the standard trials from the same sessions (Fig. 5b; Supplementary Fig. 10 , left column). Since no stimulus was presented in silent trials, the prediction was that $p_{\text {silent }}$ $=p_{A}$ (Eq. 5), because responses were all proactive, i.e. were generated from the Al process operating in isolation ("Al alone" in Fig. 5b and Supplementary Fig. 10).

For advanced/delayed trials, where the stimulus onset was advanced or delayed by $\Delta$, we first tested the prediction obtained from simply advancing or delaying the EA onset time $t_{\text {stim }}$ by $\Delta$. In a second analysis we fitted the value of the latency time $t_{E}$ and/or EA drift $v_{E}$ separately for each delay condition, leaving other parameters unchanged (Fig. $5 \mathrm{~d}-\mathrm{e}, \mathrm{g}-\mathrm{h}$; Supplementary Figs. 11 and 12, dashed blue curves, and 13).

\section{DATA CODE AVAILABILITY}

Data and code will be made available on a public repository after the manuscript is published.

\section{ACKNOWLEDGEMENTS:}


We thank Jordi Pastor for excellent discussions and Lejla Bektic and Lorena Jiménez for help with training of the animals. We thank Dani Linares, Klaus Wimmer, Anke Braun, Juan R. Castiñeiras and Alfonso Renart for helpful comments on an earlier version of the manuscript. This research was supported by the Spanish Ministry of Economy and Competitiveness together with the European Regional Development Fund (BES-2011-049131 to A.H.-M.; IJCl-2016-29358 to D.D.; SAF2015-70324-R and RTI2018-099750-B-I00 to J.R.; PSI2015-74644-JIN and RYC-2017-23231 to A.H.), the European Research Council (ERC-2015-CoG - 683209 Priors to J.R and L.H.-N.), the European Union (Marie Curie IRG PIRG07-GA-2010-268382 to J.R.).

\section{AUTHOR CONTRIBUTIONS :}

A.H.-M. and J.R. designed the first set of experiments (Group 1); A.H.-M. carried out the first set of experiments; L.H.-N., D.D., A.H. and J.R. designed the second set of experiments (Groups 2-4); D.D. carried out the second set of experiments; L.H.-N. analyzed the data; L.H.-N. and A.H. developed the generative model; all authors interpreted the data; L.H.-N., A.H. and J.R. wrote the manuscript with contributions from the rest of the authors.

\section{COMPETING INTERESTS}

The authors declare no competing interests.

\section{REFERENCES}

Becker, G. M. (1958) 'Sequential decision making: Wald's model and estimates of parameters', Journal of Experimental Psychology, pp. 628-636. doi: 10.1037/h0046519.

Blurton, S. P., Kesselmeier, M. and Gondan, M. (2012) 'Fast and accurate calculations for cumulative first-passage time distributions in Wiener diffusion models', Journal of Mathematical Psychology, pp. 470-475. doi: 10.1016/j.jmp.2012.09.002.

Bogacz, R. et al. (2006) 'The physics of optimal decision making: a formal analysis of models of performance in two-alternative forced-choice tasks', Psychological review, 113(4), pp. $700-765$.

Bogacz, R. et al. (2010) 'The neural basis of the speed-accuracy tradeoff', Trends in Neurosciences, pp. 10-16. doi: 10.1016/j.tins.2009.09.002.

Bowman, N. E., Kording, K. P. and Gottfried, J. A. (2012) 'Temporal integration of olfactory perceptual evidence in human orbitofrontal cortex', Neuron, 75(5), pp. 916-927.

van den Brink, R. L. et al. (no date) 'Temporal expectation hastens decision onset but does not affect evidence quality'. doi: 10.1101/2020.01.30.926337.

Brunton, B. W., Botvinick, M. M. and Brody, C. D. (2013) 'Rats and humans can optimally 
accumulate evidence for decision-making', Science, 340(6128), pp. 95-98.

Carpenter, R. H. and Williams, M. L. (1995) 'Neural computation of log likelihood in control of saccadic eye movements', Nature, 377(6544), pp. 59-62.

Churchland, A. K., Kiani, R. and Shadlen, M. N. (2008) 'Decision-making with multiple alternatives', Nature Neuroscience, pp. 693-702. doi: 10.1038/nn.2123.

Davis, C. M., Roma, P. G. and Hienz, R. D. (2016) 'The Rodent Psychomotor Vigilance Test (rPVT): A Method for Assessing Neurobehavioral Performance in Rats and Mice', Journal of Visualized Experiments. doi: 10.3791/54629.

Devine, C. A. et al. (2019) 'The role of premature evidence accumulation in making difficult perceptual decisions under temporal uncertainty', eLife. doi: 10.7554/elife.48526.

Ding, L. and Gold, J. I. (2013) 'The Basal Ganglia's Contributions to Perceptual Decision Making', Neuron, pp. 640-649. doi: 10.1016/j.neuron.2013.07.042.

Drugowitsch, J. et al. (2012) 'The Cost of Accumulating Evidence in Perceptual Decision Making', Journal of Neuroscience, pp. 3612-3628. doi: 10.1523/jneurosci.4010-11.2012.

Faber, L. G., Maurits, N. M. and Lorist, M. M. (2012) 'Mental fatigue affects visual selective attention', PloS one, 7(10), p. e48073.

Fisher, R. A. and Tippett, L. H. C. (1928) 'Limiting forms of the frequency distribution of the largest or smallest member of a sample', Mathematical Proceedings of the Cambridge Philosophical Society, pp. 180-190. doi: 10.1017/s0305004100015681.

Gold, J. I. and Shadlen, M. N. (2001) 'Neural computations that underlie decisions about sensory stimuli', Trends in cognitive sciences, 5(1), pp. 10-16.

Gold, J. I. and Shadlen, M. N. (2002) 'Banburismus and the brain: decoding the relationship between sensory stimuli, decisions, and reward', Neuron, 36(2), pp. 299-308.

Gold, J. I. and Shadlen, M. N. (2007) 'The Neural Basis of Decision Making', Annual Review of Neuroscience, pp. 535-574. doi: 10.1146/annurev.neuro.29.051605.113038.

Gondan, M., Blurton, S. P. and Kesselmeier, M. (2014) 'Even faster and even more accurate first-passage time densities and distributions for the Wiener diffusion model', Journal of Mathematical Psychology, pp. 20-22. doi: 10.1016/j.jmp.2014.05.002.

Good, I. J. (1979) 'Studies in the History of Probability and Statistics. XXXVII A. M. Turing's statistical work in World War Il', Biometrika, pp. 393-396. doi: 10.1093/biomet/66.2.393.

Green, D. M., Smith, A. F. and von Gierke, S. M. (1983) 'Choice reaction time with a random foreperiod', Perception \& psychophysics, 34(3), pp. 195-208.

Haith, A. M., Pakpoor, J. and Krakauer, J. W. (2016) 'Independence of Movement Preparation and Movement Initiation', The Journal of neuroscience: the official journal of the Society for Neuroscience, 36(10), pp. 3007-3015.

Hanks, T. D. et al. (2015) 'Distinct relationships of parietal and prefrontal cortices to evidence accumulation', Nature, 520(7546), pp. 220-223.

Hanks, T. D. and Summerfield, C. (2017) ‘Perceptual Decision Making in Rodents, Monkeys, 
and Humans', Neuron, pp. 15-31. doi: 10.1016/j.neuron.2016.12.003.

Hanks, T., Kiani, R. and Shadlen, M. N. (2014) 'A neural mechanism of speed-accuracy tradeoff in macaque area LIP', eLife. doi: 10.7554/elife.02260.

Hardwick, R. M. et al. (2019) 'Time-dependent competition between goal-directed and habitual response preparation', Nature human behaviour, 3(12), pp. 1252-1262.

Hermoso-Mendizabal, A. et al. (2020) 'Response outcomes gate the impact of expectations on perceptual decisions', Nature communications, 11(1), p. 1057.

Huber, P. J. (1964) 'Robust Estimation of a Location Parameter', The Annals of Mathematical Statistics, pp. 73-101. doi: 10.1214/aoms/1177703732.

Jung, K. et al. (2014) 'Bursts and heavy tails in temporal and sequential dynamics of foraging decisions', PLoS computational biology, 10(8), p. e1003759.

Kim, J. N. and Shadlen, M. N. (1999) 'Neural correlates of a decision in the dorsolateral prefrontal cortex of the macaque', Nature neuroscience, 2(2), pp. 176-185.

Lee, I. H. and Assad, J. A. (2003) 'Putaminal activity for simple reactions or self-timed movements', Journal of neurophysiology, 89(5), pp. 2528-2537.

Logue, A. W. and Peña-Correal, T. E. (1985) 'The effect of food deprivation on self-control', Behavioural Processes, pp. 355-368. doi: 10.1016/0376-6357(85)90036-1.

Maimon, G. and Assad, J. A. (2006) 'A cognitive signal for the proactive timing of action in macaque LIP', Nature neuroscience, 9(7), pp. 948-955.

Milosavljevic, M. et al. (no date) 'The Drift Diffusion Model Can Account for the Accuracy and Reaction Time of Value-Based Choices Under High and Low Time Pressure', SSRN Electronic Journal. doi: 10.2139/ssrn.1901533.

Murakami, M. et al. (2014) 'Neural antecedents of self-initiated actions in secondary motor cortex', Nature neuroscience, 17(11), pp. 1574-1582.

Murphy, P. R., Boonstra, E. and Nieuwenhuis, S. (2016) 'Global gain modulation generates time-dependent urgency during perceptual choice in humans', Nature Communications. doi: 10.1038/ncomms13526.

Navarro, D. J. and Fuss, I. G. (2009) 'Fast and accurate calculations for first-passage times in Wiener diffusion models', Journal of Mathematical Psychology, pp. 222-230. doi: 10.1016/j.jmp.2009.02.003.

Nobre, A., Correa, A. and Coull, J. (2007) 'The hazards of time', Current opinion in neurobiology, 17(4), pp. 465-470.

Pardo-Vazquez, J. L. et al. (2019) 'The mechanistic foundation of Weber's law', Nature Neuroscience, pp. 1493-1502. doi: 10.1038/s41593-019-0439-7.

Park, I. M. et al. (2014) 'Encoding and decoding in parietal cortex during sensorimotor decision-making', Nature neuroscience, 17(10), pp. 1395-1403.

Pike, A. R. (1971) 'The latencies of correct and incorrect responses in discrimination and detection tasks: Their interpretation in terms of a model based on simple counting', 
Perception \& Psychophysics, pp. 455-460. doi: 10.3758/bf03208951.

Pike, R. (1973) 'Response latency models for signal detection', Psychological Review, pp. 53-68. doi: 10.1037/h0033871.

Purcell, B. A. and Kiani, R. (2016) 'Neural Mechanisms of Post-error Adjustments of Decision Policy in Parietal Cortex', Neuron, 89(3), pp. 658-671.

Ratcliff, R. (1978) 'A theory of memory retrieval', Psychological Review, pp. 59-108. doi: 10.1037/0033-295x.85.2.59.

Ratcliff, R. et al. (2016) ‘Diffusion Decision Model: Current Issues and History', Trends in cognitive sciences, 20(4), pp. 260-281.

Ratcliff, R. and McKoon, G. (2008) 'The diffusion decision model: theory and data for two-choice decision tasks', Neural computation, 20(4), pp. 873-922.

Ratcliff, R. and Smith, P. L. (2004) 'A Comparison of Sequential Sampling Models for Two-Choice Reaction Time', Psychological Review, pp. 333-367. doi:

10.1037/0033-295x.111.2.333.

Ratcliff, R. and Tuerlinckx, F. (2002) 'Estimating parameters of the diffusion model: approaches to dealing with contaminant reaction times and parameter variability', Psychonomic bulletin \& review, 9(3), pp. 438-481.

Reddi, B. A. J. and Carpenter, R. H. S. (2000) 'The influence of urgency on decision time', Nature Neuroscience, pp. 827-830. doi: 10.1038/77739.

Reinagel, P. (2018) 'Training Rats Using Water Rewards Without Water Restriction', Frontiers in behavioral neuroscience, 12, p. 84.

Resulaj, A. et al. (2009) 'Changes of mind in decision-making', Nature, pp. 263-266. doi: 10.1038/nature08275.

Richards, J. B. et al. (1997) 'Determination of discount functions in rats with an adjusting-amount procedure', Journal of the experimental analysis of behavior, 67(3), pp. 353-366.

Roitman, J. D. and Shadlen, M. N. (2002) 'Response of neurons in the lateral intraparietal area during a combined visual discrimination reaction time task', The Journal of neuroscience: the official journal of the Society for Neuroscience, 22(21), pp. 9475-9489.

Romo, R. and Schultz, W. (1992) 'Role of primate basal ganglia and frontal cortex in the internal generation of movements. III. Neuronal activity in the supplementary motor area', Experimental brain research. Experimentelle Hirnforschung. Experimentation cerebrale, 91(3), pp. 396-407.

Salinas, E. et al. (2010) 'Waiting is the Hardest Part: Comparison of Two Computational Strategies for Performing a Compelled-Response Task', Frontiers in computational neuroscience, 4, p. 153.

Salinas, E. et al. (2014) 'Decoupling speed and accuracy in an urgent decision-making task reveals multiple contributions to their trade-off', Frontiers in Neuroscience. doi: 10.3389/fnins.2014.00085. 
Samuels, S. M. and Feller, W. (1969) 'An Introduction to Probability Theory and Its Applications, Vol. 1', Technometrics, p. 405. doi: 10.2307/1267275.

Shankar, S. et al. (2011) 'Tracking the temporal evolution of a perceptual judgment using a compelled-response task', The Journal of neuroscience: the official journal of the Society for Neuroscience, 31(23), pp. 8406-8421.

Shevinsky, C. A. and Reinagel, P. (2019) 'The Interaction Between Elapsed Time and Decision Accuracy Differs Between Humans and Rats', Frontiers in neuroscience, 13, p. 1211.

Simen, P. et al. (2009) 'Reward rate optimization in two-alternative decision making: empirical tests of theoretical predictions', Journal of experimental psychology. Human perception and performance, 35(6), pp. 1865-1897.

Simen, P. et al. (2011) 'A Model of Interval Timing by Neural Integration', Journal of Neuroscience, pp. 9238-9253. doi: 10.1523/jneurosci.3121-10.2011.

Simon, N. W. et al. (2010) 'Good things come to those who wait: attenuated discounting of delayed rewards in aged Fischer 344 rats', Neurobiology of aging, 31(5), pp. 853-862.

Smeets, J. B., Oostwoud Wijdenes, L. and Brenner, E. (2016) 'Movement Adjustments Have Short Latencies Because There is No Need to Detect Anything', Motor control, 20(2), pp. 137-148.

Stanford, T. R. et al. (2010) 'Perceptual decision making in less than 30 milliseconds', Nature Neuroscience, pp. 379-385. doi: 10.1038/nn.2485.

Steinemann, N. A., O’Connell, R. G. and Kelly, S. P. (2018) ‘Decisions are expedited through multiple neural adjustments spanning the sensorimotor hierarchy', Nature communications, 9(1), p. 3627.

Thura, D. et al. (2012) 'Decision making by urgency gating: theory and experimental support', Journal of neurophysiology, 108(11), pp. 2912-2930.

Thura, D. and Cisek, P. (2014) 'Deliberation and Commitment in the Premotor and Primary Motor Cortex during Dynamic Decision Making', Neuron, pp. 1401-1416. doi: 10.1016/j.neuron.2014.01.031.

Tweedie, M. C. K. (1945) 'Inverse Statistical Variates', Nature, pp. 453-453. doi: $10.1038 / 155453 \mathrm{a} 0$.

Vangkilde, S., Coull, J. T. and Bundesen, C. (2012) 'Great expectations: Temporal expectation modulates perceptual processing speed', Journal of Experimental Psychology: Human Perception and Performance, pp. 1183-1191. doi: 10.1037/a0026343.

Verdonck, S. and Tuerlinckx, F. (2016) 'Factoring out nondecision time in choice reaction time data: Theory and implications', Psychological review, 123(2), pp. 208-218.

Wald, A. (1947) Sequential Analysis.

Welford, A. T. (1988) 'Reaction Time, Speed of Performance, and Age', Annals of the New York Academy of Sciences, pp. 1-17. doi: 10.1111/j.1749-6632.1988.tb32958.x.

Yartsev, M. M. et al. (2018) 'Causal contribution and dynamical encoding in the striatum 
during evidence accumulation', eLife, 7. doi: 10.7554/eLife.34929. 